\title{
TumorNext: A comprehensive tumor profiling assay that incorporates high resolution copy number analysis and germline status to improve testing accuracy
}

Phillip N. Gray ${ }^{1}$, Huy Vuong ${ }^{1}$, Pei Tsai ${ }^{1}$, Hsaio-Mei Lu ${ }^{1}$, Wenbo Mu${ }^{1}$, Vickie Hsuan ${ }^{1}$, Jayne Hoo ${ }^{1}$, Swati Shah ${ }^{1}$, Lisa Uyeda ${ }^{1}$, Susanne Fox ${ }^{1}$, Harshil Patel ${ }^{1}$, Mike Janicek ${ }^{2}$, Sandra Brown ${ }^{3}$, Lavinia Dobrea ${ }^{3}$, Lawrence Wagman ${ }^{3}$, Elizabeth Plimack ${ }^{4}$, Ranee Mehra $^{4}$, Erica A. Golemis ${ }^{4}$, Marijo Bilusic ${ }^{4}$, Matthew Zibelman ${ }^{4}$, Aaron Elliott ${ }^{1}$

${ }^{1}$ Ambry Genetics, Aliso Viejo, CA, 92656, USA

${ }^{2}$ Arizona Oncology, Scottsdale, AZ, 85258, USA

${ }^{3}$ St. Joseph Health, Orange, CA, 92868, USA

${ }^{4}$ Fox Chase Cancer Center, Philadelphia, PA, 19111, USA

Correspondence to: Phillip N. Gray, email: pgray@ambrygen.com

Keywords: tumor profiling, actionable mutations, next generation sequencing, copy number variants, germline mutations

Received: June 16, $2016 \quad$ Accepted: August 26, $2016 \quad$ Published: September 08, 2016

\section{ABSTRACT}

The development of targeted therapies for both germline and somatic DNA mutations has increased the need for molecular profiling assays to determine the mutational status of specific genes. Moreover, the potential of off-label prescription of targeted therapies favors classifying tumors based on DNA alterations rather than traditional tissue pathology. Here we describe the analytical validation of a custom probe-based NGS tumor panel, TumorNext, which can detect single nucleotide variants, small insertions and deletions in 142 genes that are frequently mutated in somatic and/or germline cancers. TumorNext also detects gene fusions and structural variants, such as tandem duplications and inversions, in $\mathbf{1 5}$ frequently disrupted oncogenes and tumor suppressors. The assay uses a matched control and custom bioinformatics pipeline to differentiate between somatic and germline mutations, allowing precise variant classification. We tested 170 previously characterized samples, of which $>95 \%$ were formalin-fixed paraffin embedded tissue from 8 different cancer types, and highlight examples where lack of germline status may have led to the inappropriate prescription of therapy. We also describe the validation of the Affymetrix OncoScan platform, an array technology for high resolution copy number variant detection for use in parallel with the NGS panel that can detect single copy amplifications and hemizygous deletions. We analyzed 80 previously characterized formalin-fixed paraffin-embedded specimens and provide examples of hemizygous deletion detection in samples with known pathogenic germline mutations. Thus, the TumorNext combined approach of NGS and OncoScan potentially allows for the identification of the "second hit" in hereditary cancer patients.

\section{INTRODUCTION}

Traditional methods for tumor characterization are tumor-type specific and include assays such as immunohistochemistry (IHC), in situ hybridization (ISH), quantitative PCR (qPCR), Sanger sequencing and gene signature microarrays [1-8]. Such assays are highly specific but provide limited information. In contrast, whole genome sequencing (WGS) of tumors using next generation sequencing (NGS) is an unbiased approach that provides extensive genomic information about a tumor. Unfortunately, the cost of sequencing and associated bioinformatics handling of results is still too high for routine clinical WGS of tumor specimens. An alternative approach is exome sequencing, but even this method is not cost effective due to the large amount of data required to detect variants occurring at low frequencies (i.e. at minor allele frequencies $\leq 5 \%$ ) and time needed 
to analyze thousands of genes [13]. Targeted gene panels are currently the best option for tumor characterization as they allow multiple genes to be analyzed and can provide enough depth of coverage to detect minor allele frequencies in a cost-effective manner [9-12].

Due to the increasing popularity of NGS-based tumor testing, guidelines for detecting tumor variants have recently been established by the State of New York Health Department [13]. Based on these recommendations, samples should have enough sequence data for a minimum average coverage of 500x so that minor allele frequencies of $5 \%$ can be reliably detected. Coverage and its reliability as a quality metric can differ depending on whether amplification-based or probe-based enrichment is utilized [14]. Amplification-based NGS panels are based on the polymerase chain reaction (PCR) and are ideal when analyzing archived tissues for which material is limited. However, unless molecular barcodes are employed, the true depth of coverage cannot be determined with amplicon-based target enrichment, as PCR duplicates cannot be distinguished from amplicons generated from the original template. In addition, allele drop-out (i.e. failed primer binding due to a variant in the primer binding site that results in only one allele being amplified) resulting in false negatives is a potential concern when utilizing primer based enrichment.

In contrast to amplification-based approaches, probe-based enrichment methods use biotinylated oligonucleotide probes of up to 120 nucleotides in length designed to capture a region of interest. Since the probes are much longer than typical PCR primers, variants in the probe binding sites typically do not affect hybridization to the target region, and thus allele drop-out is not an issue. Most probe-based methods require traditional NGS library preparation in which DNA is randomly sheared, so sequence reads for a particular target will have several different start and stop coordinates. As a result, PCR duplicates can be identified and removed from the dataset, which reduces the false positive rate [15] and allows the true sequencing coverage depth to be determined.

The incorporation of a matched blood or normal tissue control from the same patient to differentiate somatic from germline mutations increases the accuracy of NGS-based tumor panels. Jones et al. published a comparison of tumor-only vs tumor/germline paired analysis and found up to one-third of actionable mutations in tumor-only analysis are classified incorrectly as somatic when they are actually germline [16]. The misclassification of variants could result in inappropriate treatment recommendations. For example, a tumor may contain a germline variant of unknown significance (VUS) in an oncogene that is predicted to be an activating mutation, but with only an intermediate confidence level. If the VUS was known to be germline, it may instead be classified as benign since inherited mutations in oncogenes are relatively rare [17]. In contrast, if the germline status of the VUS was unknown, it is more likely to be treated as a driver of disease, potentially leading to inappropriate therapy, increased health care costs and lost time for the patient. Thus, knowing the germline status of the variant can not only aid in the clinical management of the patient and their family members but also help correctly classify the variant as likely pathogenic or likely benign. Although the inclusion of incidental germline findings may complicate patient management for the oncologist [18], tumor-only analysis may lead to incorrect variant classification with possible negative impacts on patient safety and healthcare costs.

A number of clinical laboratories offering NGS panels use the data to detect copy number variations (CNVs) in addition to single nucleotide variations (SNVs), insertions and deletions (indels) and structural variants (SVs). CNV analysis of somatic specimens using data from NGS panels is limited, as such analysis cannot detect amplifications below $5 \times$ or polyploidy [10], and is restricted to genes on the NGS panel. In contrast, microarray-based platforms can provide whole genome CNV analysis and are highly sensitive, capable of detecting single copy amplifications as well as aneuploidy and polyploidy [19, 20], and have become a standard clinical laboratory test for CNV determination [21-23]. While the number of clinically actionable CNVs is currently limited, it is important to collect whole genome CNV profiles rather than a limited panel as new CNVs involved in oncogenesis may be revealed with data from greater numbers of whole genome profiles. Also, ploidy determination can have clinical significance and serve as a prognostic factor. For example, in prostate cancer, DNA aneuploidy increases with stage and grade and can serve as a prognostic indicator for hormone therapy [24, 25]. Also, aneuploidy of chromosome 17 is a prognostic indictor in breast cancer [26-28]. Although there is an added cost associated with microarray analysis, coupling NGS with a microarray provides the most accurate and reliable genomic profile of a tumor.

Here we describe the analytical validation of a custom probe-based NGS tumor panel, TumorNext, for the detection of SNVs, indels and SVs that uses a matched blood (i.e. non-tumor) control to differentiate between somatic and germline mutations. The panel targets all exons of 142 genes and select introns of 15 genes associated with solid tumors. The genes on the panel are associated with hereditary cancer or known to have clinically actionable mutations, which are genomic alterations that may predict sensitivity or resistance to standard or investigational therapies and include mutations that $(i)$ have an FDA approved therapy in the patient's tumor type; (ii) have an FDA approved therapy in a different tumor type (off-label); (iii) have a drug targeting the altered gene currently in a clinical trial; or (iv) provide prognostic information $[9,12]$. Also described is the validation of the Affymetrix OncoScan platform, a 
molecular inversion probe (MIP) array technology for $\mathrm{CNV}$ detection in FFPE specimens, intended for use in parallel with the NGS panel.

\section{RESULTS}

\section{In silico testing for bioinformatics pipeline optimization}

We conducted an in silico analysis using a targeted sequencing dataset comprised of 5,615 target regions covering approximately $3.8 \mathrm{Mb}$ and 413 genes to test the analytical sensitivity and specificity of the TumorNext bioinformatics pipeline. For each type of variant (SNV, deletion and insertion), a total of 32 datasets were simulated by combining 4 different average coverages $(100 \times, 250 \times, 500 \times$ and $1000 \times)$ and 8 different heterozygous variant frequency ranges $(0-3 \%, 3 \%-5 \%$, $5 \%-10 \%, 10 \%-20 \%, 20 \%-30 \%, 30 \%-50 \%, 50 \%-$ $80 \%, 80 \%-100 \%$ ). To test reproducibility, each dataset was simulated three times. For the simulated SNV dataset, simulations were conducted 3 times to generate $650 \mathrm{~K}$ SNVs total (Supplementary Table S1). The simulation showed that $\geq 500 \times$ coverage yielded a sensitivity of $100 \%$ and allows detection of alleles down to 3\% (Supplementary Table S2). Specificity was also $100 \%$ at $\geq 500 \times$ coverage (Supplementary Table S3). For the indel simulations, 15 different size lengths were randomly generated and binned: 1 bp, 2 bp, 3 bp, 4 bp, 5 bp, 6 bp, 7 bp, 8 bp, 9 bp, 10 bp, 11-20 bp, 21-30 bp, 31-40 bp, 41-50 bp, > 50 bp (Supplementary Table S4 for deletions and Supplementary Table S7 for insertions). For deletions, $\geq 500 \times$ coverage gave a sensitivity of $100 \%$ and allowed detection of alleles down to 3\% (Supplementary Table S5) and specificity was $100 \%$ at all coverage levels (Supplementary Table S6). For insertions, $\geq 500 \times$ coverage gave a sensitivity of $100 \%$ and allowed detection of alleles down to $3 \%$ for most insertion sizes (Supplementary Table S8). Sensitivity began to decline when insertion sizes reach $31 \mathrm{bp}$. Also, insertion detection sensitivity dropped below $100 \%$ for 7 bp insertions. Specificity was $100 \%$ at all coverage levels (Supplementary Table S9). Based on these results, all somatic samples were sequenced to a minimum average depth of coverage of 500×, which is in line with the New York Health Department guidelines.

\section{NGS panel}

A custom panel was designed to analyze 2,350 exons, 153 introns and 4 UTR regions in 142 genes associated with solid tumor cancers (Supplementary Table S10). Approximately 99 genes are frequently mutated in solid tumors and may be targets of FDA-approved or experimental therapies, and/or are prognostic indicators [29]. The remaining 43 genes are associated with hereditary cancers and the mutational status of a subset of these genes may guide treatment (i.e. BRCA1 status and PARP inhibitors). The assay consists of a hybridizationbased target enrichment and NGS. DNA is extracted from both FFPE tissue and blood and dual analysis is performed to differentiate between germline and somatic mutations. Deletion and duplication analysis, utilizing whole genome OncoScan microarray technology, is performed concurrently (Figure 1).

\section{Limit of detection}

Solid tumor samples are usually a heterogeneous mixture of cells derived from both tumor and normal tissue. Moreover, the tumor cells may consist of several subpopulations of mutant genotypes $[30,31]$ similar to a viral quasispecies [32]. As a result, allele frequencies within the tumor may occur across a broad range, with mutations of interest present at low levels (i.e. 5\%) [33]. In silico analysis using simulated datasets indicated our bioinformatics pipeline could detect SNVs and indels down to a minor allele frequency (MAF) of 3\% with 500× coverage, as described above. To evaluate the limit of detection of the TumorNext NGS panel, serial dilutions were performed with tumor samples where tumor DNA was diluted with their respective matched normal DNA to generate a range of known heterozygous allele frequencies. Sequencing coverage for indels varied from $390 \times$ to $5700 \times$. Analysis for these samples was performed using all sequence data as well as a normalized data set in which reads were removed from samples with high coverage $(>2000 \times)$ to yield an average depth of coverage of $1000 \times$ for most samples (Table 1). In the non-normalized data set, all variants were detected by the pipeline, including a c.683dupA/p.Y228* mutation in CDH1 that was detected at a MAF of $0.8 \%$ in a sample with $3187 \times$ coverage (Figure 2A). The detection of variants below 1\% was not surprising due to the high depth of coverage for this sample. For the normalized data set, the pipeline was able to detect MAFs below 5\% (Figure 2B). Coverage was more uniform for samples with SNVs (Table 2) and similar results were obtained with normalized and non-normalized samples (Figure $3 \mathrm{~A}$ and $3 \mathrm{~B}$ ) with a MAF of $1.52 \%$ detected (with $2436 \times$ coverage) in the non-normalized data set, and $2.06 \%$ detected (with $1066 \times$ coverage) in the normalized data set. Similar to what others have reported [34], the TumorNext analysis pipeline is limited by specificity rather than sensitivity. Therefore, our assay can detect variants with allele frequencies of $5 \%$ or higher with $100 \%$ concordance.

\section{Analytical sensitivity}

Four sets of samples were used to determine analytical sensitivity: HapMap samples with known genotypes (2 samples), FFPE tumor specimens previously characterized using the OncoScan hotspot panel 
(111 samples), tumor specimens previously characterized on CytoSNP850K (35 samples) and tumor specimens previously characterized by Foundation Medicine, Guardant Health or Caris (16 samples). A summary of all mutations analyzed from FFPE specimens is listed in Table 3. First, we analyzed two HapMap samples (NA10857 and NA07019) and found 91/94 calls were concordant with the reference sequence with 3 discordant calls in NA070019 (rs6685892, rs3899528 and rs619203). These 3 SNPs were Sanger sequenced and found to be concordant with the NGS data; indicating the reference genotype for these SNPs was incorrect (Supplementary Figure S1). Overall, concordance was 100\% (94/94 calls) when comparing TumorNext NGS data to the 2 HapMap sample genotypes (Supplementary Tables S11 and S12).

The OncoScan array was designed primarily to detect CNVs, but it also contains a small hot spot panel that can detect a total of 74 clinically actionable somatic mutations in 9 genes, which includes both SNVs and indels (Supplementary Table S13). A total of 111 samples were run on OncoScan and later analyzed on TumorNext (Supplementary Table S14). TumorNext detected all mutations detected by the OncoScan hotspot panel, but one mutation was inappropriately filtered out. The mutation was present in $22.66 \%$ of tumor sequence reads and $13.39 \%$ of control reads, so it was classified at a germline variant and filtered out of the somatic data. The call was PIK3CA p.H1047R/c.3140A > G, which is a wellknown oncogene mutation [35] and highly unlikely to be a germline mutation. Notably, the matched control for this specimen was tissue, whereas most other control DNA was derived from blood, and contained $15 \%$ tumor. As a result, the PIK3CA H1047R mutation was filtered out because it was present in both the tumor and matched control. While $15 \%$ tumor is high, this sample was provided to us as a non-tumor matched control and the possibility of this occurring with matched "normal" tissue with less tumor contamination (i.e. $<10 \%$ ) is a concern. As a result, our pipeline was modified to retain all calls when using tissue as a matched control. When taking all samples (111) and mutations screened (74) into account, the total number of regions analyzed was 8214 , and overall concordance was $100 \%(8214 / 8214)$.

The Illumina CytoSNP850K bead chip array contains $\sim 850,000$ SNPs spanning the entire genome with enriched coverage for 3,262 genes frequently containing copy number alterations in somatic cancer cells. The number of SNPs covered by both TumorNext and CytoSNP850K was determined to be 2,035. A total of 35 FFPE samples that were previously run on CytoSNP850K with a SNP confidence score $>95$ (n.b., Illumina recommends $>99 \%$ for non-FFPE samples) were used to determine accuracy of TumorNext. Although 138 out of the 2,035 SNPs were discordant, this reflected poor calls for only 27 SNPs in 19 genes (Supplementary Tables S15 and S16), suggesting these SNPs do not perform well on the CytoSNP850K array with FFPE samples. All discordant calls were Sanger sequenced and 134 of 135 were determined to be false positives on the CytoSNP850K array. The one discordant call was detected by TumorNext, but filtered out due to low coverage. The sample (SP11_180A14) had a depth of coverage of $587 \times$, but coverage for the discordant SNP was only $67 \times$ (below the $100 \times$ threshold for variant calling), causing it to be

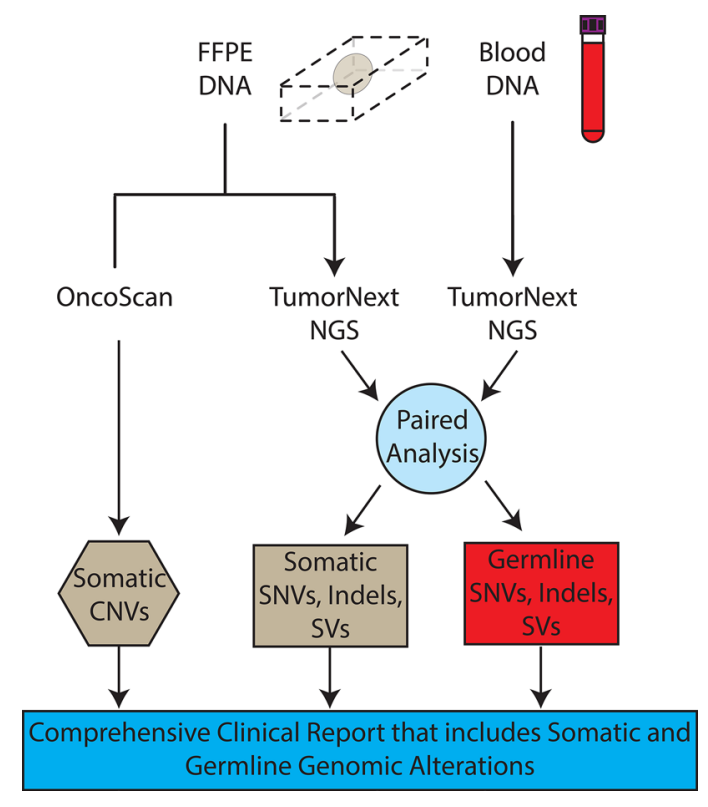

Figure 1: TumorNext workflow. DNA is extracted from both FFPE specimens and blood. FFPE DNA is analyzed using OncoScan for CNV detection and TumorNext for SNVs, indels and SVs detection. The TumorNext bioinformatics pipeline uses data from the blood sample to perform a paired analysis, which can differentiate between germline and somatic variants. The TumorNext final report provides a comprehensive analysis of somatic and germline alterations. 
Table 1: Limit of detection study: normalized coverage for samples containing known indels

\begin{tabular}{|c|c|c|c|c|c|c|c|}
\hline Sample & $\begin{array}{l}\text { Variant } \\
\text { Type }\end{array}$ & Gene Symbol & $\begin{array}{c}\text { Annotated Variant based } \\
\text { on HGVS }\end{array}$ & $\begin{array}{c}1: 2 \\
\text { Coverage }\end{array}$ & $\begin{array}{c}1: 4 \\
\text { Coverage }\end{array}$ & $\begin{array}{c}1: 8 \\
\text { Coverage }\end{array}$ & $\begin{array}{c}1: 16 \\
\text { Coverage }\end{array}$ \\
\hline \multirow[b]{2}{*}{ RD_008 } & deletion & PARP1 & c. $2275 \_2277+3$ delCAGGTA & 964 & 935 & 946 & 914 \\
\hline & deletion & HSP90AA1 & \begin{tabular}{|l|} 
c.1091_1103del13 \\
p.Y364Ffs*10
\end{tabular} & 390 & 594 & 483 & 656 \\
\hline RD_009 & deletion & BRCA1 & $\begin{array}{l}\text { c. } 3478 \_3487 \text { del10 } \\
\text { p.K1160Lfs*47 }\end{array}$ & 1489 & 1527 & 1534 & 1509 \\
\hline RD_015 & deletion & BRCA1 & $\begin{array}{l}\text { c.5101_5104delCTGA } \\
\text { p.L1701Nfs*4 }\end{array}$ & 1119 & 1130 & 1086 & 1136 \\
\hline BR13_102 & insertion & $\mathrm{CDH} 1$ & c.683dupA p.Y228* & 928 & 956 & 955 & 962 \\
\hline BR12_110 & deletion & PTEN & c.800delA p.K267Rfs*9 & 905 & 846 & 922 & 977 \\
\hline
\end{tabular}

filtered out. This particular SNP (EPHA2 rs2230597) showed up as a false positive in 3 other samples (i.e. Sanger sequencing did not confirm the call) and is located in a $\mathrm{G} / \mathrm{C}$ rich region. A total of 223 samples (including samples run multiple times) were run for the validation and the average coverage was over $1100 \times$, so this sample had below average coverage. The overall concordance was $99.94 \%(1,979 / 1,980)$ between TumorNext and CytoSNP850K array.
The third measure of accuracy consisted of comparing samples previously analyzed by Caris, Guardant Health or Foundation Medicine to results from TumorNext. A total of 29 variants (mutations and variants of unknown significance) were reported by Foundation Medicine and Guardant Health in targets covered by TumorNext. TumorNext detected 29 of 29 variants, in addition to 1 intronic variant outside Foundation's reporting range, which was Sanger confirmed. A total

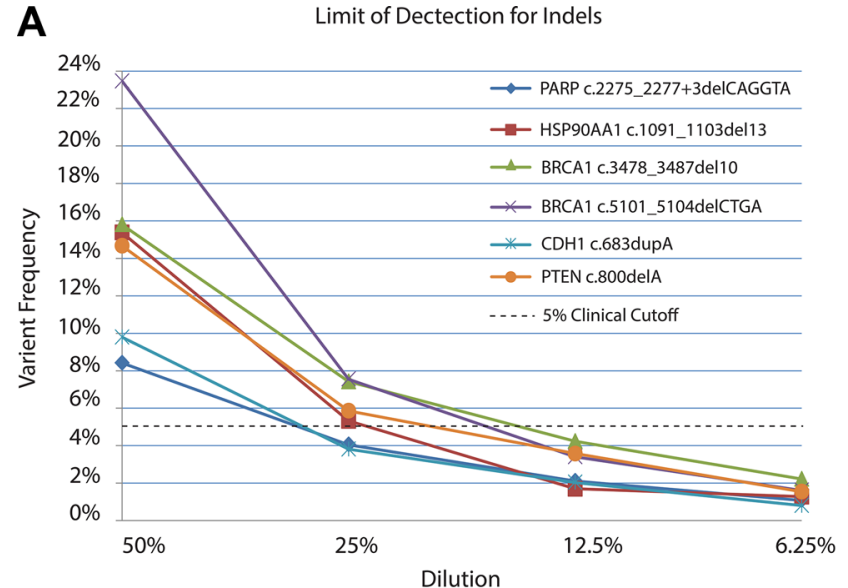

B

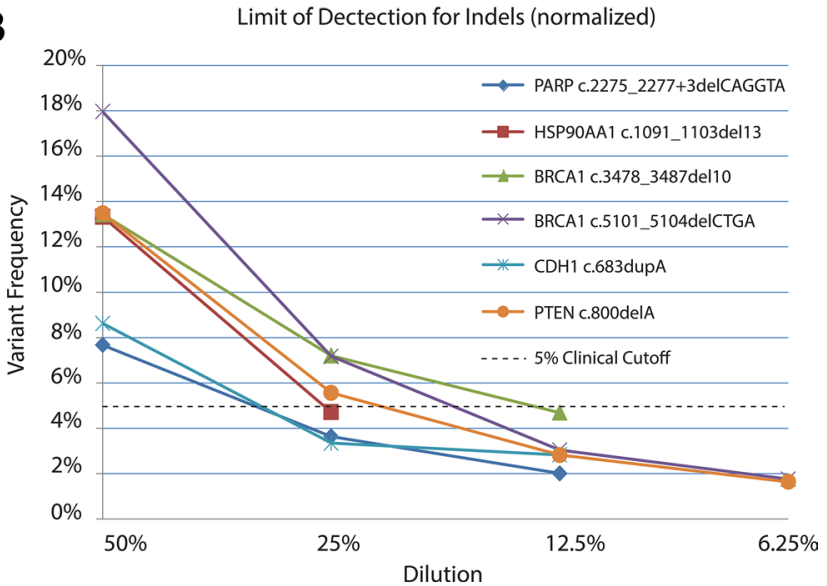

Figure 2: Limit of detection for known indels in non-normalized (A) and normalized (B) datasets. DNA extracted from tumors was serial diluted in matched control/blood DNA and samples sequenced to determine the limit of detection. 
Table 2: Limit of detection study: normalized coverage for samples containing known SNVs

\begin{tabular}{|c|c|c|c|c|c|c|c|}
\hline Sample & $\begin{array}{c}\text { Variant } \\
\text { Type }\end{array}$ & $\begin{array}{c}\text { Gene } \\
\text { Symbol }\end{array}$ & $\begin{array}{c}\text { Annotated Variant based on } \\
\text { HGVS }\end{array}$ & $\begin{array}{c}1: 2 \\
\text { Coverage }\end{array}$ & $\begin{array}{c}1: 4 \\
\text { Coverage }\end{array}$ & $\begin{array}{c}1: 8 \\
\text { Coverage }\end{array}$ & $\begin{array}{c}1: 16 \\
\text { Coverage }\end{array}$ \\
\hline BR13_184 & SNV & KRAS & c. $38 \mathrm{G}>$ A p.G13D & 1023 & 1077 & 1106 & 1072 \\
\hline BR14_75 & SNV & EGFR & c. $2573 \mathrm{~T}>$ G p.L858R & 1038 & 1047 & 1109 & 1053 \\
\hline BR13_104 & SNV & TP53 & c. $742 \mathrm{C}>\mathrm{T}$ p.R248W & 997 & 1003 & 1057 & 1045 \\
\hline BR12_110 & SNV & KRAS & c. $35 \mathrm{G}>$ A p.G12D & 1061 & 1132 & 1069 & 1127 \\
\hline BR13_10 & SNV & TP53 & c.743G > A p.R248Q & 1055 & 966 & 1029 & 1023 \\
\hline BR14_67 & SNV & TP53 & c.743G $>$ A p.R248Q & 955 & 960 & 1066 & 1019 \\
\hline
\end{tabular}

of 7 variants were reported by Caris in targets covered by TumorNext. TumorNext detected 7 of 7 variants in addition to 14 variants, which were all Sanger confirmed. Seven of these variants were on the Caris NGS panel, but not listed in their report (Table 4). Combined, a total of 51 variants were detected by TumorNext for this sample set and all concordant with either: $i$ ) results reported by the other labs, ii) Sanger confirmed variants, iii) results observed on the OncoScan hotspot panel or iv) results observed from multiple assays (i.e. Sanger and OncoScan).

In total, 2303 genotypes determined by other platforms (including novel findings that were Sanger confirmed) were compared to TumorNext and 0 false positives were detected and 1 false negative was observed. Based on these results, the analytical sensitivity (True Positives/True Positives + False Negatives) of the TumorNext panel was $2302 / 2302+1=99.96 \%$ (95\% CI, $99.76 \%-100 \%)$.

We determined some variants listed on the Foundation Medicine reports to be germline. Most of these were listed at VUSs, however, two were listed as actionable and therapy recommended. Sample RD_001_C1 had a germline mutation in ERBB2 (R896C), which was reported as actionable and treatment recommended.
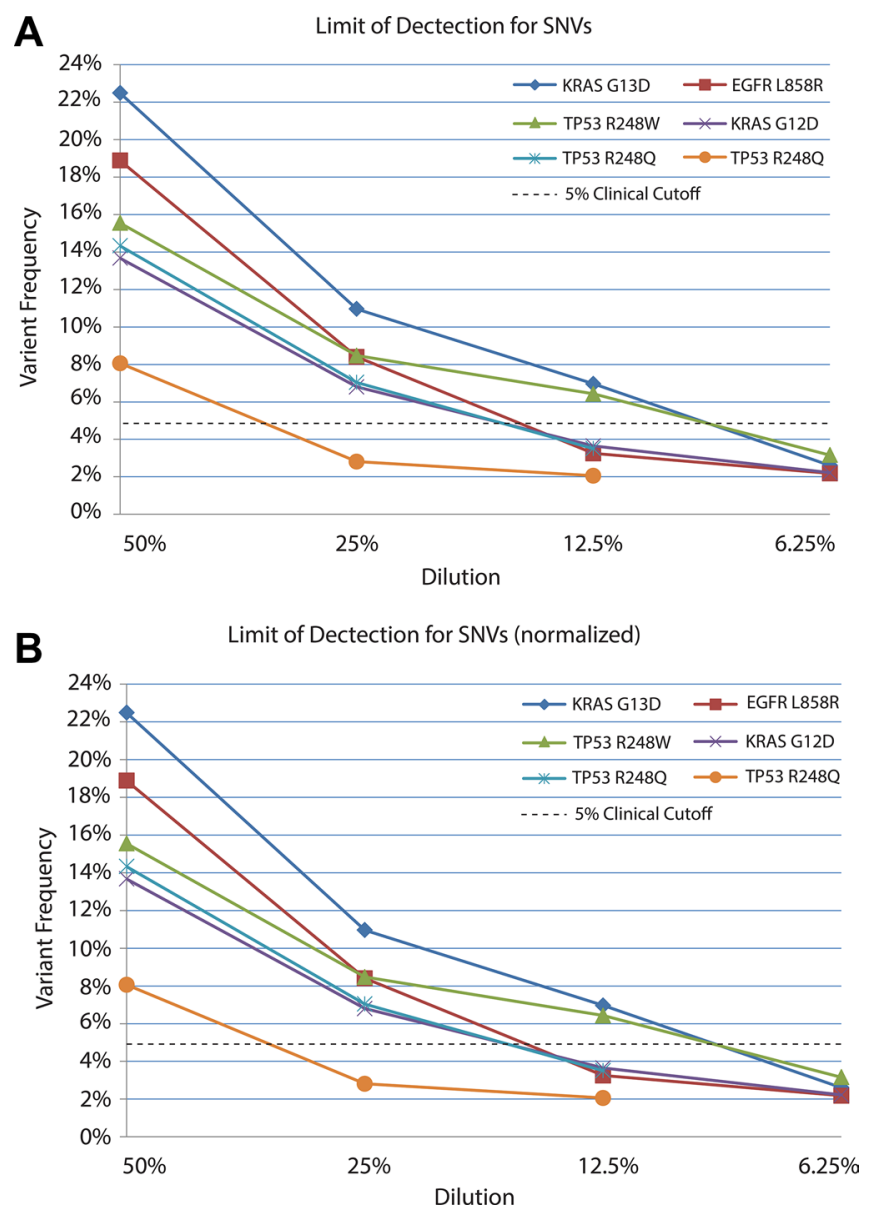

Figure 3: Limit of detection for known SNVs in non-normalized (A) and normalized (B) datasets. DNA extracted from tumors was serial diluted in matched control/blood DNA and samples sequenced to determine the limit of detection. 
Table 3: FFPE validation samples with previously known mutations

\begin{tabular}{|c|c|c|c|}
\hline Mutation Class & Gene & Mutation & Mutations Recovered \\
\hline SNV & AKT2 & p.W22* & $1 / 1$ \\
\hline SNV & APC & p.L1129S & $1 / 1$ \\
\hline SNV & ATM & p.V2424G & $1 / 1$ \\
\hline SNV & AURKB & p.R248H & $1 / 1$ \\
\hline SNV & BRAF & p.V600E & $3 / 3$ \\
\hline SNV & BRCA2 & p.F233V & $1 / 1$ \\
\hline$\overline{\mathrm{SNV}}$ & EGFR & 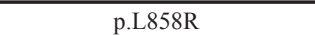 & $1 / 1$ \\
\hline SNV & EPHA5 & p.P141T & $1 / 1$ \\
\hline SNV & EPHB1 & p.F699Y & $1 / 1$ \\
\hline SNV & ERBB2 & p.R896C & $1 / 1$ \\
\hline SNV & ERBB2 & c. $575-3 \mathrm{C}>\mathrm{T}$ & $1 / 1$ \\
\hline SNV & ESR1 & p.A571V & $1 / 1$ \\
\hline SNV & FANCD2 & p.P593S & $1 / 1$ \\
\hline SNV & FGFR1 & p.S393L & $1 / 1$ \\
\hline SNV & FGFR1 & p.R822C & $1 / 1$ \\
\hline SNV & FLT3 & p.V197L & $1 / 1$ \\
\hline SNV & FLT4 & p.T810K & $1 / 1$ \\
\hline SNV & JAK3 & p.V628D & $1 / 1$ \\
\hline SNV & KRAS & p.G12D & $9 / 9$ \\
\hline SNV & KRAS & p.G12V & $4 / 4$ \\
\hline SNV & KRAS & p.G12C & $1 / 1$ \\
\hline SNV & KRAS & p.G12S & $1 / 1$ \\
\hline SNV & KRAS & p.G13D & $1 / 1$ \\
\hline SNV & KRAS & p.Q61K & $1 / 1$ \\
\hline SNV & KRAS & p.Q61H & $1 / 1$ \\
\hline SNV & MLL & p.A3247A & $1 / 1$ \\
\hline SNV & MYD88 & p.V188L & $1 / 1$ \\
\hline SNV & NF1 & p.W2494* & $1 / 1$ \\
\hline SNV & NF2 & p.R198* & $1 / 1$ \\
\hline SNV & NOTCH1 & p.G1342S & $1 / 1$ \\
\hline SNV & NRAS & p.G12D & $1 / 1$ \\
\hline SNV & NRAS & p.Q61R & $1 / 1$ \\
\hline SNV & PDGFRB & p.V316M & $1 / 1$ \\
\hline SNV & PIK3CA & p.E545K & $3 / 3$ \\
\hline SNV & PIK3CA & p.E542K & $1 / 1$ \\
\hline SNV & PIK3CA & p.H1047R & $4 / 4$ \\
\hline SNV & PIK3CG & p.R359H & $1 / 1$ \\
\hline SNV & RET & p.R77H & $1 / 1$ \\
\hline SNV & ROS1 & p.R2116I & $1 / 1$ \\
\hline SNV & TP53 & p.R282W & $1 / 1$ \\
\hline SNV & TP53 & p.R306* & $1 / 1$ \\
\hline SNV & TP53 & p.R213* & $3 / 3$ \\
\hline SNV & TP53 & p.R175H & $2 / 2$ \\
\hline SNV & TP53 & p.R248Q & $5 / 5$ \\
\hline SNV & TP53 & p.K132N & $2 / 2$ \\
\hline SNV & TP53 & p.S215G & $1 / 1$ \\
\hline SNV & TP53 & c. $782+1 \mathrm{G}>\mathrm{A}$ & $1 / 1$ \\
\hline SNV & TP53 & p.R273H & $3 / 3$ \\
\hline SNV & TP53 & p.R273C & $2 / 2$ \\
\hline SNV & TP53 & p.G245V & $1 / 1$ \\
\hline SNV & TP53 & c. $673-1 \mathrm{G}>\mathrm{C}$ & $1 / 1$ \\
\hline SNV & TP53 & p.G266V & $1 / 1$ \\
\hline SNV & TP53 & p.C275F & $1 / 1$ \\
\hline SNV & TP53 & p.Y220C & $1 / 1$ \\
\hline SNV & TSC2 & p.A196T & $1 / 1$ \\
\hline SNV & VHL & p.R3L & $1 / 1$ \\
\hline Indel & BRCA1 & p.S1655fs*16 & $2 / 2$ \\
\hline Indel & BRCA1 & p.K1160Lfs*47 & $1 / 1$ \\
\hline Indel & BRCA1 & p.L1701Nfs*4 & $1 / 1$ \\
\hline Indel & HSP90AA1 & p.Y364Ffs*10 & $1 / 1$ \\
\hline Indel & PARP1 & c.2275_2277 + 3delCAGGTA & $1 / 1$ \\
\hline Indel & PTEN & p.K267fs*9 & $1 / 1$ \\
\hline SV & BRCA1 & ins6kbEx13 duplication & $1 / 1$ \\
\hline
\end{tabular}




\section{Table 4: Concordance between TumorNext and Foundation Medicine, Guardant Health and Caris clinical reports}

\begin{tabular}{|c|c|c|}
\hline Sample & TumorNext Results & Caris, Foundation Medicine or Guardant Health \\
\hline \multirow{4}{*}{ RD_001_C1 } & ERBB2 R896C - germline & ERBB2 R896C (F) \\
\hline & BRCA1 S1655fs*16 - germline & BRCA1 S1655fs*16 (F) \\
\hline & TP53 K132N & TP53 K132N (F) \\
\hline & ESR1 A571V - germline & ESR1 A571V - VUS (F) \\
\hline \multirow{2}{*}{ RD_001_G1 } & BRCA1 S1655Yfs*16 - germline (SC) & BRCA1 S1655fs*16 (C) \\
\hline & TP53 K132N (SC) & TP53 K132N (C) \\
\hline \multirow{2}{*}{ RD_003 } & TP53 S215G (SC) & TP53 S215G (F) \\
\hline & FLT4 T810K (SC) - germline & FLT4 T810K - VUS (F) \\
\hline RD_004 & TP53 c.782+1G > A (SC) & No somatic mutations detected (C) \\
\hline \multirow{4}{*}{ RD_005 } & NRAS G12D (OS) (SC) & NRAS G12D (F) \\
\hline & PIK3CA E545K (OS) (SC) & PIK3CA E545K $(\mathrm{F})$ \\
\hline & FANCD2 P593S - germline & FANCD2 P593S - VUS (F) \\
\hline & PIK3CG R359H - germline & PIK3CG R359H - VUS (F) \\
\hline \multirow{6}{*}{ RD_007 } & EPHB1 F699Y (SC) - germline & EPHB1 F699Y - VUS (F) \\
\hline & BRCA2 F233V (SC) - germline & BRCA2 F233V - VUS (F) \\
\hline & TP53 R273H (SC) & TP53 R273H (F) \\
\hline & AURKB R248H - germline & AURKB R248H - VUS (F) \\
\hline & FGFR1 S393L, R822C - germline & FGFR1 S393L, R822C - VUS (F) \\
\hline & PDGFRB V316M - germline & PDGFRB V316M - VUS (F) \\
\hline \multirow{3}{*}{ RD_008 } & **NF1 W2494* (SC) & \multirow{3}{*}{ No somatic mutations detected (C) } \\
\hline & **PARP1 c.2275_2277+3delCAGGTA (SC) & \\
\hline & **HSP90AA1 Y364Ffs*10 (SC) & \\
\hline \multirow{5}{*}{ RD_009 } & TP53 R282W (SC) (OS) & TP53 R282W (F) \\
\hline & ERBB2 c.575-3C > T (SC) & \\
\hline & NF2 R198* (SC) & NF2 R198* (F) \\
\hline & BRCA1 K1160Lfs*47 (SC) & BRCA1 K1160Lfs*47 (F) \\
\hline & NOTCH1 G1342S - germline & NOTCH1 G1342S - VUS (F) \\
\hline \multirow{3}{*}{ RD_010 } & $* *$ MLL A3247A (SC) & \\
\hline & TP53 G245V (SC) & TP53 G245V (C) \\
\hline & APC L1129S - germline & APC L1129S - VUS (C) \\
\hline \multirow{4}{*}{ RD_012 } & **MYD88 V188L (SC) & \multirow{4}{*}{ No somatic mutations detected (C) } \\
\hline & **EPHA5 P141T (SC) & \\
\hline & FLT3 V197L (SC) & \\
\hline & TP53 c.673-1G > C (SC) & \\
\hline RD_013 & RET R77H (SC) & No somatic mutations detected (C) \\
\hline RD_014 & TP53 G266V (SC) & TP53 G266V $(\mathrm{G})$ \\
\hline \multirow{5}{*}{ RD_015 } & TP53 C275F (SC) & TP53 C275F (C) \\
\hline & BRCA1 L1701Nfs*4 (SC) & BRCA1 L1701fs (C) \\
\hline & VHL R3L (SC) & \\
\hline & JAK3 V628D (SC) & \\
\hline & **ROS1 R2116I (SC) & \\
\hline \multirow{3}{*}{ RD_016 } & TP53 Y220C (OS) & TP53 Y220C (F) \\
\hline & AKT2 W22* - germline & AKT2 W22* - VUS (F) \\
\hline & TSC2 A196T - germline & TSC2 A196T - VUS (F) \\
\hline RD_017 & ATM V2424G - germline & ATM V2424G - VUS (F) \\
\hline \multirow{5}{*}{ RD_018 } & KIT p.S123F - germline & KIT S123F (F) \\
\hline & BRCA2 K3326* - germline & BRCA2 K3326* - VUS (F) \\
\hline & TSC1 H732Y - germline & TSC1 H732Y - VUS (F) \\
\hline & \begin{tabular}{|l} 
TP53 M237I \\
\end{tabular} & TP53 M237I (F) \\
\hline & BRAF D594N & BRAF D594N (F) \\
\hline
\end{tabular}

**Gene not on Caris NGS panel

Legend: $(\mathrm{C})=$ Caris,$(\mathrm{F})=$ Foundation Medicine, $(\mathrm{G})=$ Guardant Health, $(\mathrm{OS})=$ detected on the OncoScan Hotspot panel, $(\mathrm{SC})=$ Sanger confirmed, VUS

$=$ Listed as variant of unknown significance on the Foundation One report. 
This mutation does have a COSMIC mutation Id (COSM14066) and reported as a HER2 activating mutation [36], so it is understandable why it was included in the report. However, germline mutations in oncogenes are rare, so it is highly unlikely this mutation is the driver of disease. The same observation was made for Sample RD_018, which had a germline mutation in the oncogene KIT (S123F), which also has a COSMIC mutation ID (COSM317523). Again, Foundation Medicine recommended treatment based on this mutation, but as discussed, this variant is unlikely to be a driver of disease. These examples highlight the value of determining germline status of variants for accurate classification/ assessment of contribution to disease when performing tumor profiling.

The TumorNext panel was designed to detect gene fusions and structural variants by capturing both introns and exons in 15 genes. For some genes, such as ALK, only one intron was captured as the characteristic breakpoint for the oncogenic fusion of interest (EML4-ALK) is well defined. For other genes, all introns were captured as the breakpoints for fusions of interest are unknown. For validation, the cell line $\mathrm{H} 2228$ containing the EML4-ALK fusion and a clinical specimen containing the BRCA1 exon-13 6kb duplication (Supplementary Figure S2) were used to demonstrate the panel's ability to detect fusions and structural variants. Figure 4 shows Integrative Genomics Viewer (IGV) screenshots of the EML4-ALK fusion. A total of 102 paired end reads and 118 split reads supported the inversion event in the H2228 cell line. Figure 5 shows IGV screenshots of the BRCA1 exon-13 6kb duplication from two different tumor blocks from the same patient, and two separate sequencing runs. Only paired end reads were detected in support of this tandem duplication, which numbered 4 or 5 per run. The breakpoint for this tandem duplication is located in a low complexity region containing several Alu-repeat elements, so the capture efficiency for this region is low (compatible with the low number of supporting reads).

\section{Analytical specificity}

Two of approaches were used to determine analytical specificity. First, two HapMap reference and sixteen characterized samples (from Foundation, Guardant Health or Caris) were sequenced and 0 false positive calls were made from the 411,115 bp panel (or $7,400,070$ total base pairs for 18 samples). Fourteen new variants were detected in the Caris samples, and all were Sanger confirmed. These data indicate the analytical specificity is $100 \%$, as none were false positives.

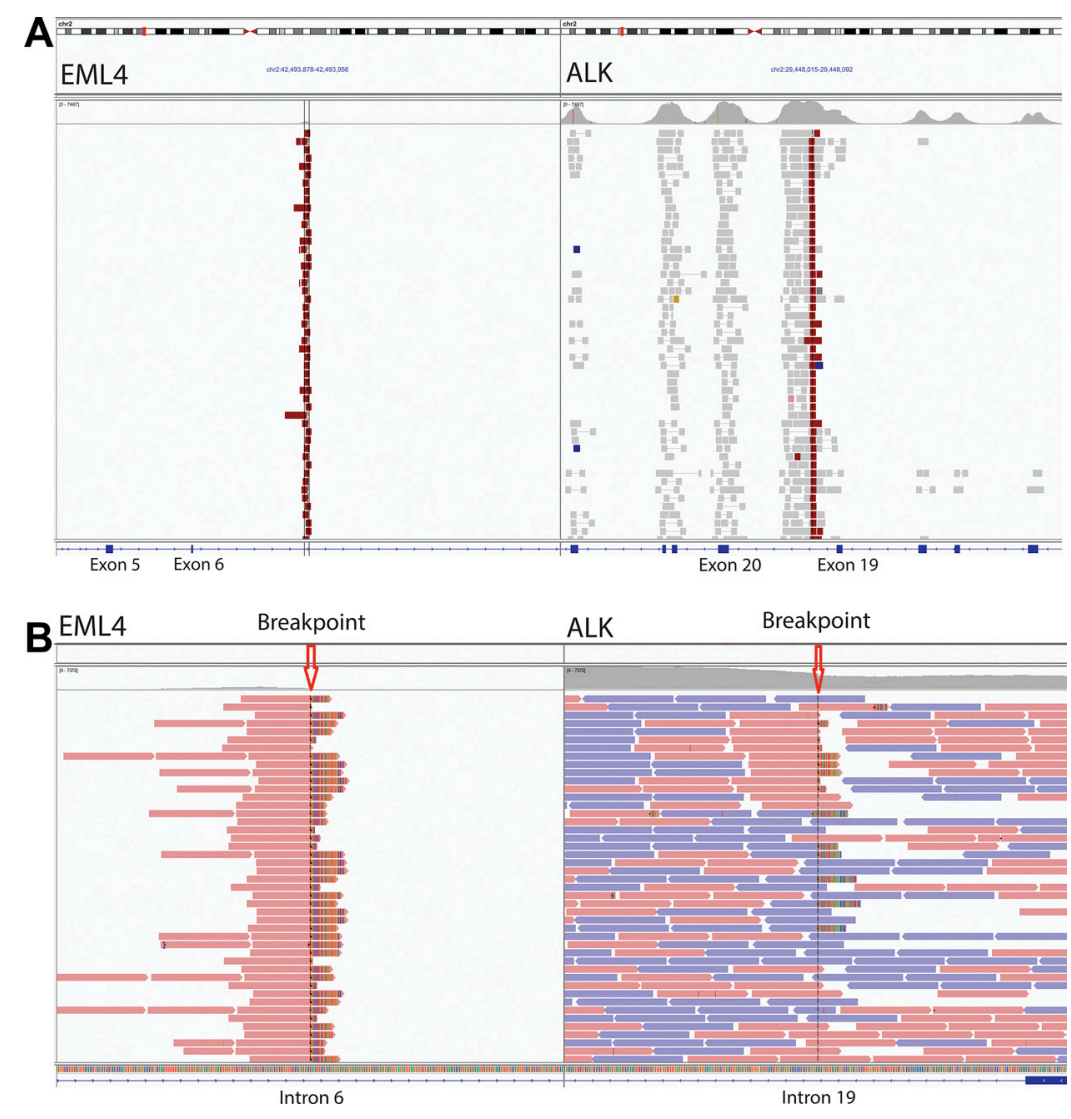

Figure 4: Gene fusion detection by TumorNext. (A) IGV screenshot of the EML4-ALK inversion showing high coverage of the breakpoint and surrounding exons in ALK. (B) Zoomed in screenshot showing the breakpoints and several split reads that support the fusion (Note: the multi-colored part of the read aligns to the fusion partner). 
Second, data from 8214 sites genotyped by OncoScan (111 samples $\times 74$ mutations analyzed) were concordant with the TumorNext NGS data. Of these sites, 8164 were true negatives and there were no false positives, so the analytical specificity (True Negatives/True Negatives + False Positives) is $8164 / 8164+0=100 \%$ (95\% CI, 99.95\%-100\%).

\section{Precision and reproducibility}

Seven samples with Sanger-confirmed somatic mutations were used to assess intra- and interreproducibility. Mutations included point mutations, deletions and a gene fusion. Samples were assayed in triplicate as intra- (1 sample) or inter-run replicates (6 samples) and were prepared separately by different technicians on multiple dates using non-redundant barcodes to minimize potential barcode bias. All known variants were detected in each run, with the majority detected at similar frequencies and normalized coverage across replicates (Table 5). The reproducibility of coverage was also assessed by determining the percent of bases detected $\geq 100 \times$ across all 27 validation runs for exons only, and for the total capture region (Figure 6). As expected, the percentage of bases with $\geq 100 \times$ coverage was higher for exons, as exon targets typically are higher complexity sequences and are not typically associated with increased off-target sequence (which reduces overall target specificity and coverage). In contrast, introns are comprised mostly of low complexity sequences and are associated with higher off-target sequence when enriched with probe-based capture methods.

\section{OncoScan to detect gross abnormalities}

The Affymetrix OncoScan system is a microarraybased platform that utilizes molecular inversion probes (MIPs), a target enrichment technology that functions via capture by circularization [37]. This process works by designing a single stranded DNA probe that has
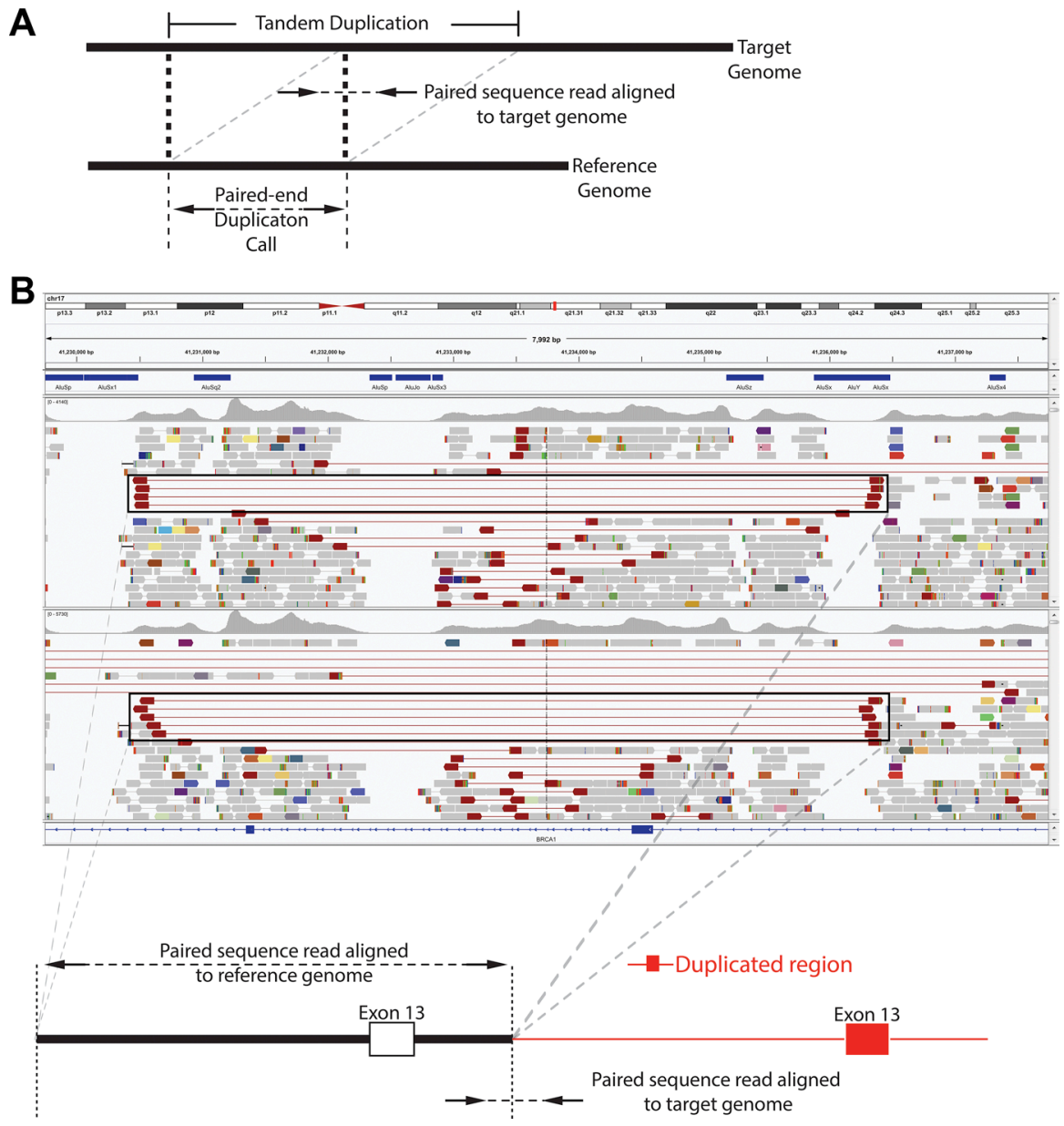

Figure 5: Tandem duplication detection by TumorNext. (A) Schematic of tandem duplication detection using paired end reads. Reads are represented by arrows. Standard paired end reads face each other as represented in the target genome alignment. A tandem duplication will result in paired end reads facing away from each other when aligned to a reference genome. (B) IGV screenshot of two tumor specimens with a known BRCA1 exon-13 $6 \mathrm{~kb}$ duplication and schematic of the duplication. The supporting paired end reads are located inside the boxes. 
Table 5: Results of reproducibility study for TumorNext

\begin{tabular}{|c|c|c|c|c|c|c|c|c|}
\hline Sample & Known Variant & Run & Barcode & $\begin{array}{c}\text { Sample } \\
\text { Coverage }\end{array}$ & $\begin{array}{l}\text { Variant } \\
\text { Called? }\end{array}$ & $\begin{array}{c}\text { Variant } \\
\text { Coverage }\end{array}$ & $\begin{array}{c}\text { Variant } \\
\text { Frequency }\end{array}$ & $\begin{array}{c}\text { Normalized } \\
\text { Coverage }\end{array}$ \\
\hline \multirow{9}{*}{ RD_006 } & \multirow{3}{*}{$\begin{array}{l}\text { KRAS p.G12D } \\
\text { NM_033360 } \\
\text { c.35G>A }\end{array}$} & 1 & B03 & 2187 & Yes & 2615 & $35.45 \%$ & 1.20 \\
\hline & & 2 & B01 & 1492 & Yes & 1474 & $28.85 \%$ & 0.99 \\
\hline & & 3 & B09 & 2622 & Yes & 3122 & $31.62 \%$ & 1.19 \\
\hline & \multirow{3}{*}{$\begin{array}{l}\text { NF1 p.A132G } \\
\text { NM_001042492 } \\
\text { c.395C }>\text { G } \\
\end{array}$} & 1 & $\mathrm{~B} 03$ & 2187 & Yes & 2391 & $33.71 \%$ & 1.09 \\
\hline & & 2 & B01 & 1492 & Yes & 1506 & $29.97 \%$ & 1.01 \\
\hline & & 3 & B09 & 2622 & Yes & 2787 & $32.05 \%$ & 1.06 \\
\hline & \multirow{3}{*}{$\begin{array}{l}\text { BRAF p.G464V } \\
\text { NM_004333 } \\
\text { c. } 1391 \mathrm{G}>\mathrm{T}\end{array}$} & 1 & B03 & 2187 & Yes & 2297 & $35.50 \%$ & 1.05 \\
\hline & & 2 & B01 & 1492 & Yes & 1799 & $30.91 \%$ & 1.21 \\
\hline & & 3 & B09 & 2622 & Yes & 3217 & $35 \%$ & 1.23 \\
\hline \multirow{9}{*}{ RD_007 } & \multirow{3}{*}{$\begin{array}{l}\text { BRCA2 p.F233V } \\
\text { NM_000059 } \\
\text { c.697T }>\mathrm{G}\end{array}$} & 1 & B04 & 742 & Yes & 815 & $34.97 \%$ & 1.10 \\
\hline & & 2 & B02 & 294 & Yes & 345 & $30.72 \%$ & 1.17 \\
\hline & & 3 & $\mathrm{~B} 10$ & 1420 & Yes & 1732 & $33.25 \%$ & 1.22 \\
\hline & \multirow{3}{*}{$\begin{array}{l}\text { TP53 p.R273H } \\
\text { NM_000546 } \\
\text { c. } 818 \mathrm{G}>\mathrm{A}\end{array}$} & 1 & B04 & 742 & Yes & 461 & $68.11 \%$ & 0.62 \\
\hline & & 2 & B02 & 294 & Yes & 291 & $69.66 \%$ & 0.99 \\
\hline & & 3 & $\mathrm{~B} 10$ & 1420 & Yes & 1186 & $70.35 \%$ & 0.84 \\
\hline & \multirow{3}{*}{$\begin{array}{l}\text { EPHB1 p.F699Y } \\
\text { NM_004441 } \\
\text { c.2096T }>\text { A }\end{array}$} & 1 & B04 & 742 & Yes & 1018 & $23.99 \%$ & 1.37 \\
\hline & & 2 & B02 & 294 & Yes & 465 & $24.24 \%$ & 1.58 \\
\hline & & 3 & $\mathrm{~B} 10$ & 1420 & Yes & 1970 & $22.19 \%$ & 1.39 \\
\hline \multirow{9}{*}{ RD_008 } & \multirow{3}{*}{$\begin{array}{l}\text { PARP1 } \\
\text { NM_001618 c.2275_2277+3delCAGGTA }\end{array}$} & 1 & B08 & 1710 & Yes & 1725 & $35.50 \%$ & 1.01 \\
\hline & & 2 & $\mathrm{~B} 03$ & 397 & Yes & 416 & $27.16 \%$ & 1.05 \\
\hline & & 3 & B11 & 917 & Yes & 884 & $30.67 \%$ & 0.96 \\
\hline & \multirow{3}{*}{$\begin{array}{l}\text { HSP90AA1 p.Y364Ffs*10 NM_005348 } \\
\text { c.1091_1103del13 }\end{array}$} & 1 & B08 & 1710 & Yes & 1913 & $29.41 \%$ & 1.12 \\
\hline & & 2 & B03 & 397 & Yes & 242 & $16.12 \%$ & 0.61 \\
\hline & & 3 & B11 & 917 & Yes & 906 & $20.94 \%$ & 0.99 \\
\hline & \multirow{3}{*}{$\begin{array}{l}\text { NF1 p.W2494* } \\
\text { NM_001042492 } \\
\text { c.7482G }>\text { A } \\
\end{array}$} & 1 & B08 & 1710 & Yes & 870 & $65.44 \%$ & 0.51 \\
\hline & & 2 & B03 & 397 & Yes & 143 & $52.82 \%$ & 0.36 \\
\hline & & 3 & B11 & 917 & Yes & 436 & $59.95 \%$ & 0.48 \\
\hline \multirow{12}{*}{ RD_009 } & \multirow{6}{*}{$\begin{array}{l}\text { ERBB2 } \\
\text { NM_004448 } \\
\text { c.575-3C >T } \\
\text { BRCA1 p.K1160Lfs*47 NM_007294 } \\
\text { c.3478_3487del10 }\end{array}$} & 1 & B09 & 1423 & Yes & 888 & $50.96 \%$ & 0.62 \\
\hline & & 2 & B04 & 1162 & Yes & 623 & $48.48 \%$ & 0.54 \\
\hline & & 3 & B19 & 1989 & Yes & 1232 & $44.32 \%$ & 0.62 \\
\hline & & 1 & B09 & 1423 & Yes & 1567 & $41.86 \%$ & 1.10 \\
\hline & & 2 & B04 & 1162 & Yes & 1423 & $38.89 \%$ & 1.22 \\
\hline & & 3 & B19 & 1989 & Yes & 2363 & $39.62 \%$ & 1.19 \\
\hline & \multirow{3}{*}{$\begin{array}{l}\text { TP53 p.R282W } \\
\text { NM_000546 } \\
\text { c.844C }>\text { T } \\
\end{array}$} & 1 & B09 & 1423 & Yes & 713 & $56.68 \%$ & 0.50 \\
\hline & & 2 & B04 & 1162 & Yes & 829 & $48.55 \%$ & 0.71 \\
\hline & & 3 & B19 & 1989 & Yes & 1217 & $49.12 \%$ & 0.61 \\
\hline & NF2 p.R198* & 1 & B09 & 1423 & Yes & 965 & $48.70 \%$ & 0.68 \\
\hline & NM_000268 & 2 & B04 & 1162 & Yes & 753 & $38.18 \%$ & 0.65 \\
\hline & c. $592 \mathrm{C}>\mathrm{T}$ & 3 & B19 & 1989 & Yes & 1334 & $44.83 \%$ & 0.67 \\
\hline \multirow{6}{*}{ RD_010 } & \multirow{3}{*}{$\begin{array}{l}\text { MLL p.A3247A } \\
\text { NM_001197104 } \\
\text { c.9741C }>\text { T }\end{array}$} & 1 & $\mathrm{~B} 10$ & 1035 & Yes & 1308 & $85.87 \%$ & 1.26 \\
\hline & & 2 & B08 & 504 & Yes & 544 & $83.43 \%$ & 1.08 \\
\hline & & 3 & $\mathrm{~B} 20$ & 695 & Yes & 788 & $86.83 \%$ & 1.13 \\
\hline & TP53 p.G245V & 1 & $\mathrm{~B} 10$ & 1035 & Yes & 554 & $82.31 \%$ & 0.54 \\
\hline & NM_000546 & 2 & B08 & 504 & Yes & 383 & $85.04 \%$ & 0.76 \\
\hline & & 3 & $\mathrm{~B} 20$ & 695 & Yes & 370 & $86.62 \%$ & 0.53 \\
\hline & EML4 (intron 6)-ALK fusion (intron 19) & 1 & $\mathrm{~B} 21$ & 5428 & Yes & 934 & $* \mathrm{~N} / \mathrm{A}$ & 0.17 \\
\hline H2228 & chr2:29448092>chr2:42493956 & 2 & $\mathrm{~B} 02$ & 4129 & Yes & 756 & $* \mathrm{~N} / \mathrm{A}$ & 0.18 \\
\hline & & 3 & $\mathrm{~B} 19$ & 5672 & Yes & 1051 & $* \mathrm{~N} / \mathrm{A}$ & 0.19 \\
\hline & NRAS p.G12D & 4 & $\mathrm{~B} 20, \mathrm{~B} 22, \mathrm{~B} 25$ & 1317 & Yes & 1399 & $89.34 \%$ & 1.06 \\
\hline & NM_002524 & 4 & B20,B22,B25 & 1783 & Yes & 1975 & $88.29 \%$ & 1.11 \\
\hline & c. $35 \mathrm{G}>\mathrm{A}$ & 4 & $\mathrm{~B} 20, \mathrm{~B} 22, \mathrm{~B} 25$ & 1141 & Yes & 1297 & $86.31 \%$ & 1.14 \\
\hline KD_005 & PIK3CA p.E545K & 4 & $\mathrm{~B} 20, \mathrm{~B} 22, \mathrm{~B} 25$ & 1317 & Yes & 1035 & $32.21 \%$ & 0.79 \\
\hline & NM_006218 & 4 & B20,B22,B25 & 1783 & Yes & 1232 & $31.11 \%$ & 0.69 \\
\hline & & 4 & $\mathrm{~B} 20, \mathrm{~B} 22, \mathrm{~B} 25$ & 1141 & Yes & 921 & $30.84 \%$ & 0.81 \\
\hline
\end{tabular}

*Frequency cannot be determined for structural variants using DELLY. 
complementary regions to a genomic target at its $5^{\prime}$ and $3^{\prime}$ ends. The complementary regions hybridize to the target leaving a single nucleotide gap (typically a SNP) between the ends that is subsequently filled in, resulting in a circularized probe. Non-reactive probes and genomic DNA are removed by exonuclease treatment. The circularized probes are processed further and hybridized to the OncoScan array for CNV analysis. The main advantage of this technology is the fact the sample DNA is only used for the initial MIP hybridization. All subsequent steps use the MIPs that successfully bound to their target region. As a result, the assay is not as sensitive to DNA quality as other methods, making it ideal for FFPE specimens. The platform is very robust and its utility has been demonstrated in over 140 publications.

\section{Analytical sensitivity}

The analytical sensitivity of OncoScan was determined using FFPE specimens previously characterized by ISH, IHC, qPCR, CytoSNP850K and NGS. First, a total of 30 breast and 9 ovarian cancer specimens with known HER2/neu status were analyzed on OncoScan (Table 6). The HER2/neu status was determined by either FISH (23 samples; 3 equivocal), IHC (7 samples), Oncotype DX (2 samples), a qPCR assay offered by Genomic Health (Redwood City, CA), or CISH (7 samples). All IHC and Oncotype DX results were negative (i.e. not amplified) and matched the OncoScan data. There were some discordant calls between the ISH data and OncoScan; however, some of the discrepancies may be due to the limitations inherent in ISH assays. FISH and CISH scoring is calculated by comparing the ratio of HER2 (ERBB2) probe signals to probes targeting the centromeric region of chromosome 17 (CEP17), as well as the average
HER2 probe signals per cell. Per ASCO guidelines for duel probe analysis [38], ratios $<2.0$ with an average HER 2 copy number $<4.0$ signals per cell indicate a nonamplification. Ratios $<2.0$ with an average HER2 $\geq 4.0$ and $<6.0$ are equivocal. To classify as HER2-positive, three scenarios are possible for dual probes: 1) ratios $\geq 2.0$ with an average HER2 copy number $\geq 4.0,2$ ) ratios $\geq 2.0$ with an average HER2 copy number $<4.0$ and 3 ) ratios $<2.0$ with an average HER2 copy number $\geq 6.0$ [38]. It is well known the HER2/CEP17 ratio can be skewed if the copy number of the CEP17 region is altered [39, 40]. For example, if CEP17 is co-amplified with HER, no difference will be observed in the HER2/CEP17 ratio.

A total of 30 specimens with known HER2/neu status determined by FISH or CISH were analyzed on OncoScan. Three of these specimens (H2N8, H2N17 and $\mathrm{H} 2 \mathrm{~N} 23$ ) were classified as equivocal due to an average HER 2 spot count of $\geq 4.0$ and $<6.0$ and HER2/CEP17 ratio of $<2$.0. OncoScan determined 2 of these specimens to be unamplified $(\mathrm{CN}=2)$ and 1 amplified $(\mathrm{CN}=4)$. The amplified specimen contained a CEP17 amplification, which will skew the HER2/CEP17 ratio. With the HER2 spot count at 4.9 , this sample would easily be classified as Her2+ with a corrected HER2/CEP17 ratio. The other 2 samples did not contain CEP17 amplifications, and their HER2/CEP17 ratios match the reported OncoScan copy number $(\mathrm{CN}=2)$. The average HER2 spot count was determined to be greater than 4 for both samples, which highlights a drawback from using ISH for determining HER2/neu status. ISH analysis only evaluates a limited number of cells (40 cells) and HER2 status may vary in different regions of the analyzed tumor section and throughout the specimen due to heterogeneity. In contrast, OncoScan analyzes DNA from $80 \mathrm{ng}$ of DNA, which equates to $\sim 13 \mathrm{~K}$ diploid cells (1ng of DNA contains DNA

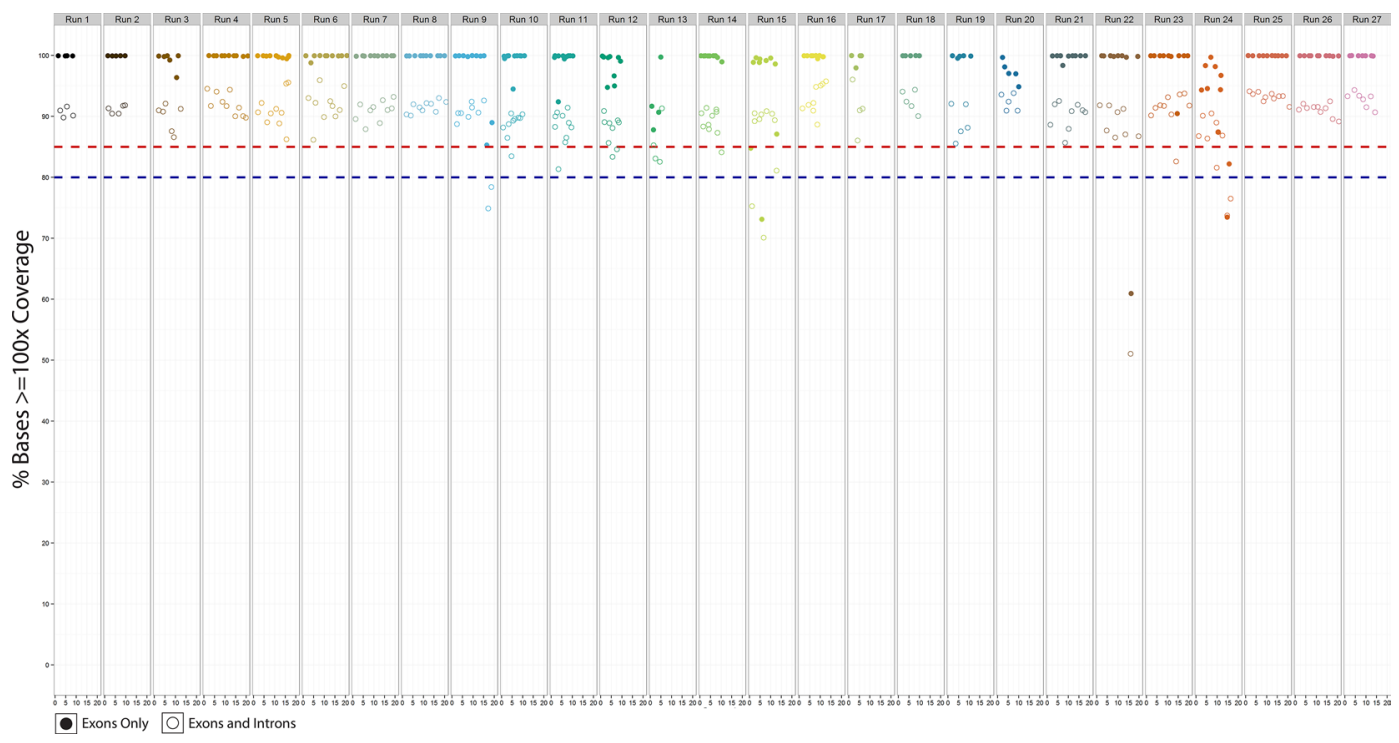

Figure 6: The percent bases covered for target exons only (solid circles) and target exons and introns (hollow circles) for all 27 validation runs. 
Table 6: HER2/neu status: comparison of oncoscan to previously characterized samples

\begin{tabular}{|c|c|c|c|}
\hline \multirow{2}{*}{ Specimen ID } & HER2/neu Status & \multirow{2}{*}{ Average HER2 spot count } & \multirow{2}{*}{ OncoScan Result } \\
\hline & FISH Result (ratio) & & \\
\hline $\mathrm{H} 2 \mathrm{~N} 1$ & Unamplified (1.2) & 2.6 & $2 n$ \\
\hline $\mathrm{H} 2 \mathrm{~N} 2$ & Unamplified (0.7) & 1.9 & $2 n$ \\
\hline $\mathrm{H} 2 \mathrm{~N} 3$ & Unamplified (1.3) & 2.4 & $2 n$ \\
\hline $\mathrm{H} 2 \mathrm{~N} 4$ & Unamplified (0.9) & 1.7 & $1.67 n^{* *}$ \\
\hline $\mathrm{H} 2 \mathrm{~N} 5$ & Unamplified (1.2) & 3.1 & $2 n$ \\
\hline H2N6 & Unamplified (1.2) & 2.3 & $2 n$ \\
\hline $\mathrm{H} 2 \mathrm{~N} 7$ & Unamplified (1.2) & 2 & $2 n$ \\
\hline $\mathrm{H} 2 \mathrm{~N} 8$ & Equivocal (1.5) & 4.4 & $2 n$ \\
\hline $\mathrm{H} 2 \mathrm{~N} 9$ & Amplified (2.4) & 6.5 & $4 n$ \\
\hline $\mathrm{H} 2 \mathrm{~N} 10$ & Amplified (2.0) & 4.3 & $3 n$ \\
\hline $\mathrm{H} 2 \mathrm{~N} 11$ & Amplified (1.2) & 6 & $2.33 n * *$ \\
\hline $\mathrm{H} 2 \mathrm{~N} 12$ & Unamplified (1.1) & 1.6 & $2 n$ \\
\hline $\mathrm{H} 2 \mathrm{~N} 13$ & Unamplified (1.1) & 3 & $3 n^{*}$ \\
\hline $\mathrm{H} 2 \mathrm{~N} 14$ & Unamplified (1.1) & 3.1 & $4 n^{*}$ \\
\hline $\mathrm{H} 2 \mathrm{~N} 15$ & Unamplified (1.1) & 2.9 & $2 n$ \\
\hline $\mathrm{H} 2 \mathrm{~N} 16$ & Unamplified (1.1) & 3 & $2 n$ \\
\hline $\mathrm{H} 2 \mathrm{~N} 17$ & Equivocal (1.4) & 4.1 & $2 n$ \\
\hline $\mathrm{H} 2 \mathrm{~N} 18$ & Amplified (2.4) & 5 & $4 n$ \\
\hline H2N19 & Unamplified (1.1) & 3.5 & $2 n$ \\
\hline $\mathrm{H} 2 \mathrm{~N} 20$ & Unamplified (1.6) & 3.4 & $4 n^{*}$ \\
\hline $\mathrm{H} 2 \mathrm{~N} 21$ & Unamplified (1.1) & 3.3 & $4 n^{*}$ \\
\hline $\mathrm{H} 2 \mathrm{~N} 22$ & Amplified (2.1) & 3.3 & $6 n$ \\
\hline \multirow[t]{2}{*}{$\mathrm{H} 2 \mathrm{~N} 23$} & Equivocal (1.9) & 4.9 & $4 n *$ \\
\hline & CISH Results (ratio) & & \\
\hline $\mathrm{H} 2 \mathrm{~N} 24$ & Unamplified (0.94) & 1.8 & $2 n$ \\
\hline $\mathrm{H} 2 \mathrm{~N} 25$ & Unamplified (1.2) & 2.1 & $2 \mathrm{n}$ \\
\hline $\mathrm{H} 2 \mathrm{~N} 26$ & Unamplified (1.03) & 1.85 & 1n \\
\hline $\mathrm{H} 2 \mathrm{~N} 27$ & Unamplified (1.09) & 1.8 & $2 n$ \\
\hline $\mathrm{H} 2 \mathrm{~N} 28$ & Unamplified (1.1) & 2.2 & $2 n$ \\
\hline $\mathrm{H} 2 \mathrm{~N} 29$ & Unamplified (1.03) & 1.95 & $3 n$ \\
\hline \multirow[t]{2}{*}{$\mathrm{H} 2 \mathrm{~N} 30$} & Unamplified (1.16) & 1.85 & $2 \mathrm{n}$ \\
\hline & IHC Results & & \\
\hline $\mathrm{H} 2 \mathrm{~N} 31$ & Negative & N/A & $2 n$ \\
\hline $\mathrm{H} 2 \mathrm{~N} 32$ & Negative & N/A & $2 \mathrm{n}$ \\
\hline $\mathrm{H} 2 \mathrm{~N} 33$ & Negative & N/A & $2 \mathrm{n}$ \\
\hline $\mathrm{H} 2 \mathrm{~N} 34$ & Negative & N/A & $2 n$ \\
\hline $\mathrm{H} 2 \mathrm{~N} 35$ & Negative & N/A & $2 n$ \\
\hline $\mathrm{H} 2 \mathrm{~N} 36$ & Negative & N/A & $2 n$ \\
\hline \multirow[t]{2}{*}{$\mathrm{H} 2 \mathrm{~N} 37$} & Negative & N/A & $2 \mathrm{n}$ \\
\hline & Oncotype DX Results & & \\
\hline $\mathrm{H} 2 \mathrm{~N} 38$ & Negative & N/A & $2 n$ \\
\hline $\mathrm{H} 2 \mathrm{~N} 39$ & Negative & N/A & $1 \mathrm{n}$ \\
\hline
\end{tabular}

*Sample has CEP17 amplification.

**Note: The copy number reported by OncoScan is the average copy number for aberrant/tumor cells only. If the TuScan software cannot determine \% aberrant cells/tumor, the copy number reported will be from the total cell population and will be a non-integer (i.e. 1.67, 2.33, etc.). 
from 167 diploid cells). Moreover, ISH analysis can be biased as a pathologist may focus on an area with high staining, whereas OncoScan provides data from the entire specimen. Sample H2N11 also highlights this issue as it was classified as HER2/neu amplified solely from the average HER2 spot count. OncoScan determined this sample to be highly heterogeneous with HER2 amplified $(\mathrm{CN}=2.33)$ in only a minority of cells. This was a case where the HER2 spot count determination was biased due to the region of the specimen evaluated.

There were 4 additional specimens with CEP17 amplifications and these were classified as unamplified (i.e. HER2-negative). OncoScan detected a copy number of 4 for three of the specimens, suggesting these samples were HER2-positive and the HER2 amplification was masked due to the CEP17 amplification. OncoScan detected a copy number of 3 for the fourth sample (H2N13), which is a single copy amplification; the CEP17 amplification may have also masked this amplification as specimen H2N10 is classified as amplified (HER2/ CEP17 ratio $=2.0$ and HER2 spot count $=4.3$ ), and also contains a copy number of 3 as determined by OncoScan. One specimen (H2N29) that was classified as unamplified based on $\mathrm{CISH}$, OncoScan reported as $\mathrm{CN}=3$. This sample did not contain a CEP17 amplification and may represent a false positive. Conversely, this sample may be true positive, but CISH failed to detect the HER2 amplification due to the limited number of cells analyzed.

A second set of samples (6 total) that had been previously characterized by Foundation Medicine or Guardant Health were also analyzed for accuracy. Foundation Medicine and Guardant Health use NGS data to detect CNVs; Foundation Medicine can detect CNVs for any gene on their panel, whereas Guardant analyzes 16 out of 68 genes for CNVs (as of March 2015). Table 7 summarizes the results from OncoScan, Foundation Medicine, and Guardant Health. A total of 24 genes were listed in Foundation Medicine reports as having CNVs and 1 gene was listed in a Guardant Health report. OncoScan produced the same result for 23/25 instances. The discordant calls include:

- The MCL1 gene in sample RD001_T_C1 OncoScan did not report a deletion in this region as the probe density is very low ( 2 probes within a $2 \mathrm{Mb}$ region of the gene). Moreover, OncoScan did not detect any CNVs in the adjacent regions.

- The ATM gene in sample RD001_T_C1 Foundation reported a homozygous deletion for exons 1-28, whereas OncoScan detected copy neutral loss (LOH in the diploid state) for exons 1-59.

A total of 35 FFPE samples that were previously characterized on the Illumina CytoSNP850K array with a SNP confidence score $>95$ were used to determine accuracy of OncoScan. Unlike OncoScan, the CytoSNP850K array was not designed specifically to analyze FFPE samples. Rather than analyze all regions throughout the genome, we restricted the accuracy study to 98 genes that are considered clinically actionable (Supplementary Table S17). Based on the CytoSNP850K data, a total of 25 samples had a CNV in at least one of the 98 genes. OncoScan detected 199 of 201 CNVs, however, there were a total of 72 discordant calls based on classification of the CNV (i.e. CN Gain, CN Loss or $\mathrm{LOH}$ ). We observed the majority of CNVs occurred in large regions $(>10 \mathrm{Mb})$, so all discordant calls were easily evaluated by analyzing the $\mathrm{B}$ allele frequency (BAF) and $\log -\mathrm{R}$ ratio $(\log \mathrm{R})$ plots (Note: A distinction was not made between "Copy Number Gain" and "High Copy Number Gain" calls, so a "Copy Number Gain" call in one platform was considered concordant with "High Copy Number Gain" in the other platform). With the exception of 1 call, all discordant calls were found to be concordant after review of the BAF and $\log$ R plots. Examples of resolved discordant calls are included in Supplementary Table S18. In one sample, half the target (ATM gene) was deleted and the other half amplified. In this case, OncoScan classified the event as CN Loss and CytoSNP850K classified as CN Gain.

Concordance was determined by dividing the number of common calls by the total calls (number of genes analyzed) and was $99 \%$ or higher for all samples (Table 8). The one discordant call that was not resolved by analysis of BAF and $\log$ plots includes a slight amplification of the CDK2NA gene in sample RD_008. The OncoScan $\log \mathrm{R}$ and BAF plots show LOH, but no amplification. The Genome Studio plots show a slight amplification and $\mathrm{LOH}$ in this region.

In total, $263 \mathrm{CNVs}$ determined by other platforms were compared to OncoScan and 2 false negatives (both in sample RD_001_T_C1) were observed. Based on these results (and excluding equivocal FISH cases), the analytical sensitivity (True Positives/True Positives + False Negatives) of the TumorNext panel is $257 / 257+2=$ 99.2\% (95\% CI, 97.24\%-99.91\%).

\section{Analytical specificity}

The analytical specificity (i.e. the false positive rate) is determined by dividing the true negatives (TN) by the sum of the false positives (FP) and true negatives $(\mathrm{TN} / \mathrm{FP}+\mathrm{TN})$. We estimated the analytical specificity of OncoScan using the concordance data from the HER2/ neu study. A total of 29 true negatives (i.e. unamplified) were confirmed with OncoScan with 1 false positive call (H2N29). Overall, the analytical specificity is $29 / 1+29=$ $96.7 \%$ (95\% CI, 82.8\% - 99.9\%).

We were unable to use the data from the Foundation Medicine reports to calculate the analytical specificity due to the lack of true negatives (i.e. their assay cannot detect amplifications below 5 copies, so the absence of a call for a particular gene does not mean it is $2 n$ ). This highlights the challenge of validating a more sensitive 
Table 7: OncoScan CNV result comparison to clinical reports from Foundation Medicine and Guardant Health

\begin{tabular}{|c|c|c|c|c|}
\hline Sample & Gene & OncoScan Results & $\begin{array}{l}\text { Foundation Medicine or } \\
\text { Guardant Health Result }\end{array}$ & Concordant \\
\hline \multirow{4}{*}{ RD_001_T_C1 } & MYC & Amp & $\operatorname{Amp}(\mathrm{F})$ & Yes \\
\hline & ATM & $\begin{array}{l}\text { Copy Neutral Loss in region containing } \\
\text { exons } 1-59\end{array}$ & Del exons 1-28 (F) & No \\
\hline & KLHL6 & Amp & $\operatorname{Amp}(\mathrm{F})$ & Yes \\
\hline & MCL1 & $\begin{array}{l}\text { No Change (only } 2 \text { probes in } 2 \mathrm{Mb} \\
\text { region of gene) }\end{array}$ & $\operatorname{Del}(\mathrm{F})$ & No \\
\hline \multirow{7}{*}{ RD_003_T } & BCL6 & Amp & $\operatorname{Amp}(\mathrm{F})$ & Yes \\
\hline & KLHL6 & Amp & $\operatorname{Amp}(\mathrm{F})$ & Yes \\
\hline & PIK3CA & Amp & $\operatorname{Amp}(\mathrm{F})$ & Yes \\
\hline & TERC & Amp & $\operatorname{Amp}(\mathrm{F})$ & Yes \\
\hline & PRKCI & Amp & $\operatorname{Amp}(\mathrm{F})$ & Yes \\
\hline & QKI & Amp & $\operatorname{Amp}(\mathrm{F})$ & Yes \\
\hline & PARK2 & Amp & $\operatorname{Amp}(\mathrm{F})$ & Yes \\
\hline \multirow{7}{*}{ RD_007_T } & EGFR & Amp & $\operatorname{Amp}(\mathrm{F})$ & Yes \\
\hline & ERBB2 & Amp & $\operatorname{Amp}(\mathrm{F})$ & Yes \\
\hline & CCNE1 & Amp & $\operatorname{Amp}(\mathrm{F})$ & Yes \\
\hline & CEBPA & Amp & $\operatorname{Amp}(\mathrm{F})$ & Yes \\
\hline & FGF10 & Amp & $\operatorname{Amp}(\mathrm{F})$ & Yes \\
\hline & IL7R & Amp & $\operatorname{Amp}(\mathrm{F})$ & Yes \\
\hline & RICTOR & Amp & $\operatorname{Amp}(\mathrm{F})$ & Yes \\
\hline \multirow{2}{*}{ RD_009_T } & MYC & Amp & $\operatorname{Amp}(\mathrm{F})$ & Yes \\
\hline & SDHA & Amp & $\operatorname{Amp}(\mathrm{F})$ & Yes \\
\hline \multirow{4}{*}{ RD_017_T } & MITF & Amp & $\operatorname{Amp}(\mathrm{F})$ & Yes \\
\hline & FOXP1 & Amp & Amp (F) & Yes \\
\hline & IKZF1 & Del & $\operatorname{Del}(\mathrm{F})$ & Yes \\
\hline & EGFR & Del & $\operatorname{Del}(\mathrm{F})$ & Yes \\
\hline RD_014_T & PIK3CA & Amp & $\operatorname{Amp}(\mathrm{G})$ & Yes \\
\hline
\end{tabular}

Legend: Amp = amplification, Del = deletion, $(\mathrm{F})=$ Foundation Medicine and $(\mathrm{G})=$ Guardant Health.

platform as OncoScan can detect more CNVs than NGS methods. Moreover, OncoScan provides whole genome CNV status, whereas NGS panels are limited to the number of genes analyzed. While the number of clinically actionable genes based on CNVs is limited, relying on a panel for CNV determination can produce misleading results. This is illustrated in sample RD 017, which exhibits extensive polyploidy (Supplementary Figure S3). Most NGS bioinformatic pipelines include differences in read depth as a mechanism to determine CNV status. In this specimen, almost the entire genome was amplified uniformly to $4 \mathrm{n}$, so there are only a small number of regions where read depth differences can be distinguished. Thus, using an NGS based approach for CNV analysis in polyploidy samples may yield misleading results. Practically every gene was amplified in this specimen and the FoundationOne report only lists 2 (both classified as equivocal); MITF and FOXP1, which are located in the same region of chromosome 3 with a copy number of $6 \mathrm{n}$. A region containing the genes ZNF217, AURKA, GNAS, which are on the FoundationOne panel, was also amplified to $6 \mathrm{n}$ but not reported. This is probably due to the fact MITF and FOXP1 are flanked by regions at a lower copy number, whereas the amplified fragment containing ZNF217, AURKA and GNAS is located at the end of the chromosome arm and only flanked by one region at a lower copy number (Supplementary Figure S3).

Samples RD_003 and RD_007 also exhibited extensive polyploidy and have several amplifications $\geq 5 \times$ that were not reported by Foundation Medicine. With the exception of ERBB2 (HER2), all focal amplifications detected by NGS appear to be limited to regions surrounded 
Table 8: OncoScan concordance for 98 actionable genes

\begin{tabular}{|c|c|c|c|c|}
\hline Sample & Number of Genes Analyzed & $\begin{array}{c}\text { Number of Altered } \\
\text { Genes }\end{array}$ & $\begin{array}{l}\text { Discordant } \\
\text { Calls }\end{array}$ & Concordance $(\%)^{* *}$ \\
\hline RD_002 & 98 & 5 & 0 & 100 \\
\hline RD_005A1 & 98 & 9 & 0 & 100 \\
\hline RD_008 & 98 & 17 & 1 & 99 \\
\hline 1002837_84444 & 98 & 4 & 0 & 100 \\
\hline 1005934 & 98 & 5 & 0 & 100 \\
\hline $1006138 \_169167$ & 98 & 19 & 0 & 100 \\
\hline 1006807_182985 & 98 & 12 & 0 & 100 \\
\hline $1008712-139858$ & 98 & 10 & 0 & 100 \\
\hline $1009749-251424$ & 98 & 3 & 0 & 100 \\
\hline $1009953-256930$ & 98 & 4 & 0 & 100 \\
\hline 1011142 & 98 & 1 & 0 & 100 \\
\hline $1012045-334325$ & 98 & 7 & 0 & 100 \\
\hline $1012374 \_346270$ & 98 & 1 & 0 & 100 \\
\hline $1013301-375808$ & 98 & 9 & 0 & 100 \\
\hline 1013490-384009 & 98 & 12 & 0 & 100 \\
\hline $1014032-395561$ & 98 & 9 & 0 & 100 \\
\hline $1014378-402958$ & 98 & 3 & 0 & 100 \\
\hline $1014577-406010$ & 98 & 2 & 0 & 100 \\
\hline $3000642-384350$ & 98 & 5 & 0 & 100 \\
\hline $3000643-383734$ & 98 & 8 & 0 & 100 \\
\hline BR14-194 05-01T & 98 & 29 & 0 & 100 \\
\hline S005-1060-1C-54417 & 98 & 8 & 0 & 100 \\
\hline S09-259 & 98 & 3 & 0 & 100 \\
\hline SP11-180 A14 & 98 & 14 & 0 & 100 \\
\hline Total & & 199 & 1 & $99.5 \%$ \\
\hline
\end{tabular}

Average Concordance (does not include 10 samples w/o calls in the 98 genes).

by a dramatic drop in sequencing coverage (Supplementary Figures S4 and S5). OncoScan is capable of detecting more subtle amplifications. For example, RD_007 contains focal amplifications in IDH2, AXIN1, ARFRP1 and SOCS1, clinically actionable genes on Foundation's panel, but the copy number difference to the surrounding areas may be too small for NGS to detect (Supplementary Figure S5). Interestingly, ARFRP1 was recently found to be co-amplified with cycling genes (CCND1, CCND2, CCND3 and CCNE1) and this sample also contains a CCNE1 amplification at $33 \times$ [41]. Also, FGF10, IL7R and RICTOR are all reported as CNVs by Foundation Medicine, but SDHA is part of the same amplification (at a copy number of 7 on chromosome 5) and not reported. This may be due to its proximity to the region where a drop in coverage is observed, which is several Mbs away (Supplementary Figure S4). Sample RD_003 contains focal amplifications in RAD51B (partial), VHL, KIT, KDR, PDRGFRA and FANCD2 at a copy number of 4 and PRSS8 amplification at a copy number of 5, all of which are below the level of detection for NGS methods (Supplementary Figure S5), so were not reported by Foundation Medicine. A CDKN1A focal amplification is also present in this sample at a copy number of 6 and was not reported (Supplementary Figure S5).

Another advantage of using OncoScan rather than NGS methods for CNV detection in somatic tumors is the ability to detect hemizygous mutations. For hereditary cancer patients, the so-called "second hit" described in the two-hit hypothesis[42] often times is loss of heterozygosity with the mutant allele in a hemizygous state in the tumor, driving disease [43]. The detection of hemizygous deletions in somatic tumors using NGS methods has been demonstrated using whole exome sequencing of tumor and matched blood [44], but remains challenging for gene panels. The clinical significance of detecting hemizygous mutants is illustrated in a recent publication by Mateo el al., which showed response to olaparib (a PARP inhibitor) in a patient with a somatic 
hemizygous deletion in both BRCA2 and PALB2 [45], homologous recombination (HR) DNA repair genes. Sample RD_001_C1 has a germline mutation in BRCA1 (S1655fs*16) at $49 \%$ frequency (in $80 \%$ tumor) in the NGS data and hemizygous deletion (i.e. LOH) in BRCA1, suggesting the mutant allele is present in a hemizygous state in the tumor and driver of disease. OncoScan also revealed RD_009 has hemizygous deletions in ATM, CHEK1, CHEK2, BRCA1, BRCA2, BRIP1, RAD51C and RAD51D (Figure 7), which represents 8 of the 13 genes involved in HR DNA repair [46] and suggests this patient is a candidate for PARP inhibitor treatment. These deletions were not reported by Foundation Medicine. This patient also has a somatic frameshift mutation in BRCA1 (p.K1160Lfs*47) at 32\% in the NGS data (in 50\% tumor), suggesting this allele is present in the tumor in a hemizygous state.

\section{Precision and reproducibility}

OncoScan provides a genome-wide CNV profile, but only a limited number of genes (approximately 98) are considered clinically actionable. For the precision and reproducibility study, we restricted analysis to reported CNVs in these 98 clinically actionable genes. Samples were prepared separately by different technicians on different dates and ran in triplicate as inter-assay replicates (5 samples) and intra-assay (1 sample) replicates. Concordance ranged from $95 \%$ to $100 \%$ for the interassay reproducibility study with lowest concordance observed in 1003748_120089, a specimen with increased heterogeneity (Table 9). The CNV events varied slightly each time a sampling of the DNA pool was taken, resulting in slightly reduced reproducibility. Concordance was $100 \%$ for intra-assay reproducibility (Table 10 ).

\section{DISCUSSION}

The ability to classify tumors based on DNA alterations rather than traditional tissue pathology is changing the paradigm of cancer treatment into a more personalized approach. As more targeted therapies are developed and if basket trials show patient benefit from "off-label" use of drugs, there will be an increased need for comprehensive tumor profiling as more patients will be prescribed therapy based on DNA mutations. TumorNext was developed to detect actionable mutations with the added benefit of determining whether a mutation is either somatic or germline. Our validation highlighted cases where knowing the germline status of variants was critical for accurate variant classification. The assay leverages both probe-based target enrichment coupled with NGS for SNV, indel and SV detection, and incorporates use of OncoScan for global CNV detection. Our decision to incorporate this technology stems from the recent observation by Ciriello et al. that showed a bias in the type of mutations found in specific tumor types, such that specific tumors contained primarily either CNVs or SNVs/indels, but not both [47]. Breast and ovarian cancers displayed the largest percentage of CNVs in the Ciriello study and make up the vast majority of specimens analyzed at Ambry Genetics. Moreover, OncoScan provides hemizygous deletion status, which is often times the "second hit" for patients with germline mutations.

Most of the CNVs detected in the validation samples, including HER2-postive samples, consisted of either whole or partial (i.e. quarter to half) chromosome copy number changes and most samples had an excessive number of CNVs. As a result, the clinical reporting of CNVs is restricted to genes listed in Supplementary Table S17 as well as all HR DNA repair genes. All $\mathrm{CNVs}$ reported by OncoScan for these genes are manually reviewed. Moreover, deletions (both homo and hemizygous) are only reported for tumor suppressor and DNA repair genes, and only focal amplifications greater than 3 are reported for oncogenes. However, this brings into question the ISH HER2-postive cases that were compared to OncoScan as only 2 of the 5 positive cases contained a focal amplification. The other 3 specimens were classified as HER2-positive based on ISH, but the ERBB2 amplifications were part of a large event (Figure 8). One possible explanation for the difference may be that the assignment of these cases as HER2-positve may not be correct since ERBB2 was not selectively amplified.

TumorNext has been launched in our clinical lab and initial results have identified actionable germline and somatic mutations ( $\mathrm{SNV}$, CNVs and gene fusions) for most cases submitted. Ambry has decided to follow the ACMG recommendations for incidental findings and automatically reports germline findings in 20 genes on TumorNext that fall within the ACMG gene list [48]. Ambry's client base primarily consists of treating physicians that routinely order germline testing, so we have not encountered a situation where the treating physician does not want to know the germline status of the ACMG genes. Patients who do not want to know their germline status are eligible for TumorNext, but this will require the treating physician to withhold the germline results. Also, unlike Ambry's germline tests, such as CancerNext, germline findings identified by TumorNext are not Sanger confirmed and only germline variants classified as pathogenic and likely pathogenic are included on the TumorNext report. The TAT is $2-3$ weeks, which is comparable to other somatic tests on the market.

The described validation was conducted primarily with FFPE tumor blocks and slides using approximately $50 \mathrm{uM}$ of tissue at $>20 \%$ tumor tissue. This amount of tissue was sufficient to yield a minimum of $500-1000 \mathrm{ng}$ of DNA, which is the optimal DNA input for NGS library prep for bait-capture protocols, so this was established as tissue requirements for TumorNext. DNA extracted from 
Table 9: Results for the inter-assay reproducibility study

\begin{tabular}{|c|c|c|c|c|}
\hline Sample No & $\begin{array}{c}\text { (Val 1) } \\
\text { DATES: } 10 / 31 / 14, \\
\text { 11/7/14, 12/1/14 and } \\
\text { 12/10/14 } \\
\text { Technician } 1\end{array}$ & $\begin{array}{c}\text { Precision Study } \\
\text { (Val 2) } \\
\text { DATE: } 12 / 11 / 14,1 / 23 / 15, \\
2 / 10 / 15 \\
\text { Technician 2 }\end{array}$ & $\begin{array}{c}\text { Reproducibility Study } \\
\text { (Val 3) } \\
\text { DATE:1/27/15 } \\
\text { Technician } 1\end{array}$ & $\begin{array}{c}\text { Overall } \\
\text { Concordance }\end{array}$ \\
\hline 10-SU4610-B2 & 73/74 CNVs & 74/74 CNVs & 72/74 CNVs & $99 \%$ \\
\hline 1003748_120089 & $21 / 22 \mathrm{CNVs}$ & 21/22 CNVs & $21 / 22 \mathrm{CNVs}$ & $95 \% *$ \\
\hline 1006138_169167 & $16 / 16 \mathrm{CNVs}$ & 16/16 CNVs & 16/16 CNVs & $100 \%$ \\
\hline 1013214_374406 & $82 / 82 \mathrm{CNVs}$ & $82 / 82$ CNVs & 81/82 CNVs & $99 \%$ \\
\hline 1013301_375808 & $13 / 13 \mathrm{CNVs}$ & 13/13 CNVs & $12 / 13 \mathrm{CNVs}$ & $97 \%$ \\
\hline
\end{tabular}

*\% Tumor could not be determined for this sample, indicating increased heterogeneity. The CNV events varied slightly each time a sampling of the DNA pool was taken, resulting in slightly reduced reproducibility.

Table 10: Results for the intra-assay reproducibility study

\begin{tabular}{|c|c|c|c|c|}
\hline \multicolumn{1}{c}{ Sample No } & $\begin{array}{l}\text { Date: 2/11/15 } \\
\text { Technician 1 }\end{array}$ & \multicolumn{1}{c}{$\begin{array}{c}\text { Date: 2/11/15 } \\
\text { Technician 1 }\end{array}$} & \multicolumn{1}{c}{$\begin{array}{c}\text { Date: 2/11/15 } \\
\text { Technician 1 }\end{array}$} & $\begin{array}{c}\text { Overall } \\
\text { Concordance }\end{array}$ \\
\hline $1006138 \_169167$ & $16 / 16 \mathrm{CNVs}$ & $16 / 16 \mathrm{CNVs}$ & $16 / 16 \mathrm{CNVs}$ & $100 \%$ \\
\hline
\end{tabular}

core-needle biopsies has been analyzed with TumorNext using 100ng input, but the assay was not optimized for low DNA input. Only a portion of the DNA added to a reaction is analyzed as not all DNA fragments are converted successfully to the NGS library (i.e. not all input DNA undergoes successful ligation and amplification). Moreover, DNA extracted from FFPE somatic tumors is heterogeneous and will have varying degrees of damage that may limit amplification. It is essential that sufficient DNA input is used to compensate for the DNA damage and heterogeneity in FFPE specimens to provide an unbiased, representative sampling of the total DNA. The limited amount of DNA isolated from core-needle biopsies can exacerbate this problem. As a result, we are developing a separate protocol for low input FFPE samples to limit sampling bias.
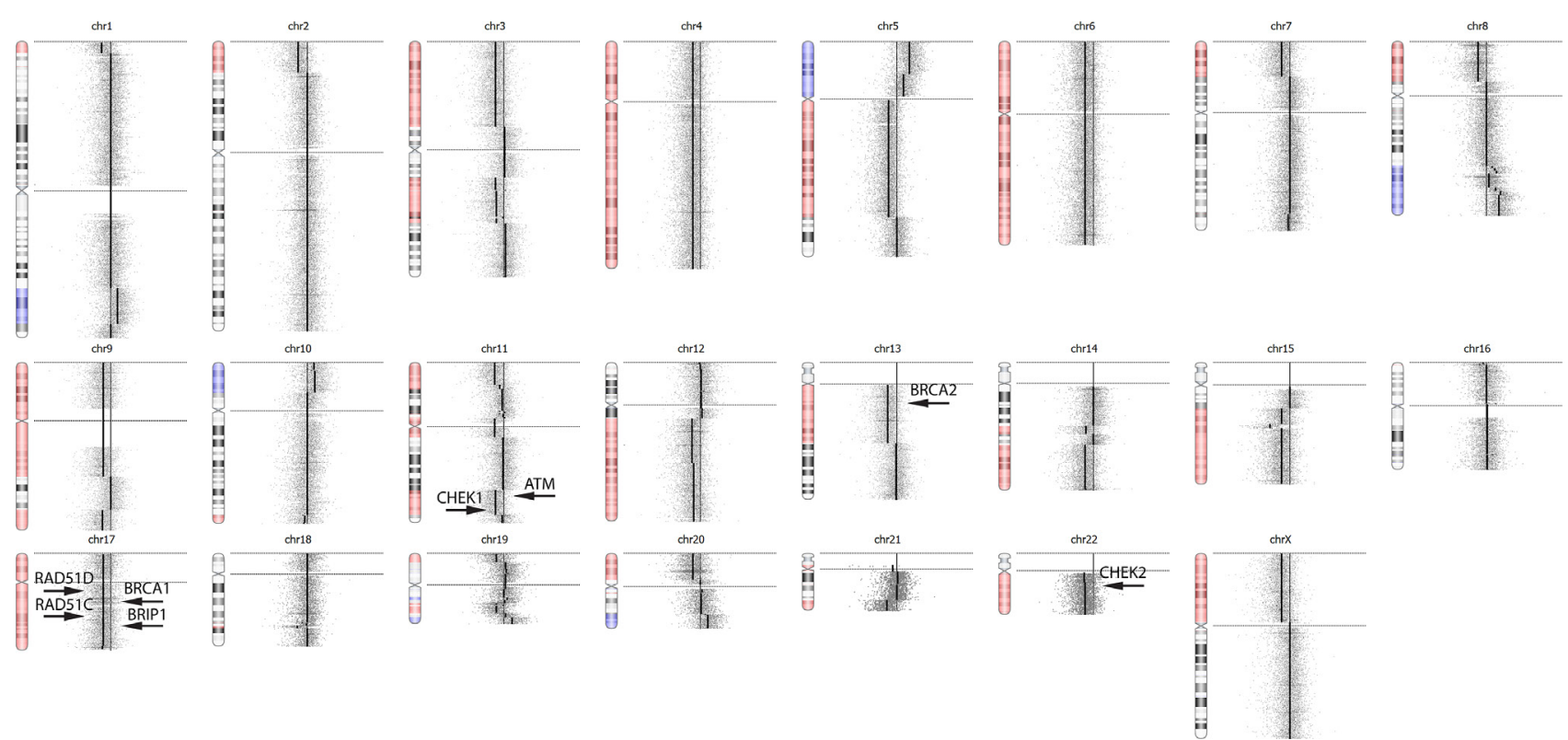

Figure 7: OncoScan Result for RD_009. OncoScan revealed RD_009 has hemizygous deletions in ATM, CHEK1, CHEK2, BRCA1, BRCA2, BRIP1, RAD51C and RAD51D, which represents 8 of the $\overline{1} 3$ genes involved in homologous recombination DNA repair and suggests this patient is a candidate for PARP inhibitor treatment. These hemizygous deletions were not detected using NGS methods for CNV analysis. 


\section{MATERIALS AND METHODS}

\section{Validation samples}

For the validation, two types of samples were used: 1) HapMap DNA samples and 2) genomic DNA isolated from FFPE tissue (tumor and normal) from the following cancer types: breast, ovarian, uterine, colon, kidney, liver, melanoma, head and neck. All FFPE specimens had been previously characterized by either Illumina CytoSNP850K array, Sanger Sequencing, NGS panel (FoundationOne ${ }^{\mathrm{TM}}$, Guardant Health or Caris), OncoScan hotspot panel, FISH, CISH, IHC, qPCR or multiple methods. In addition, a matched blood sample was included when available. All FFPE specimens were reviewed by a pathologist and contained $\geq 20 \%$ tumor cellularity.

\section{NGS gene panel design and capture protocol}

A custom panel was designed to analyze 2,350 exons, 153 introns and 4 UTR regions in 142 genes associated with solid tumor cancers (Supplementary Table S18). Approximately 99 genes are frequently mutated in somatic solid tumors and may be targets of FDA-approved or experimental therapies, or prognostic indicators. The remaining 43 genes are associated with hereditary cancers and the mutational status of a subset of these genes may guide treatment (i.e. BRCA1 status and PARP inhibitors). The assay consists of a hybridization-based capture/target enrichment and sequence analysis using massively parallel sequencing. The panel is composed of 9,564 biotinylated xGen Lockdown probes synthesized by Integrated DNA Technologies (IDT, Coralville, IA).
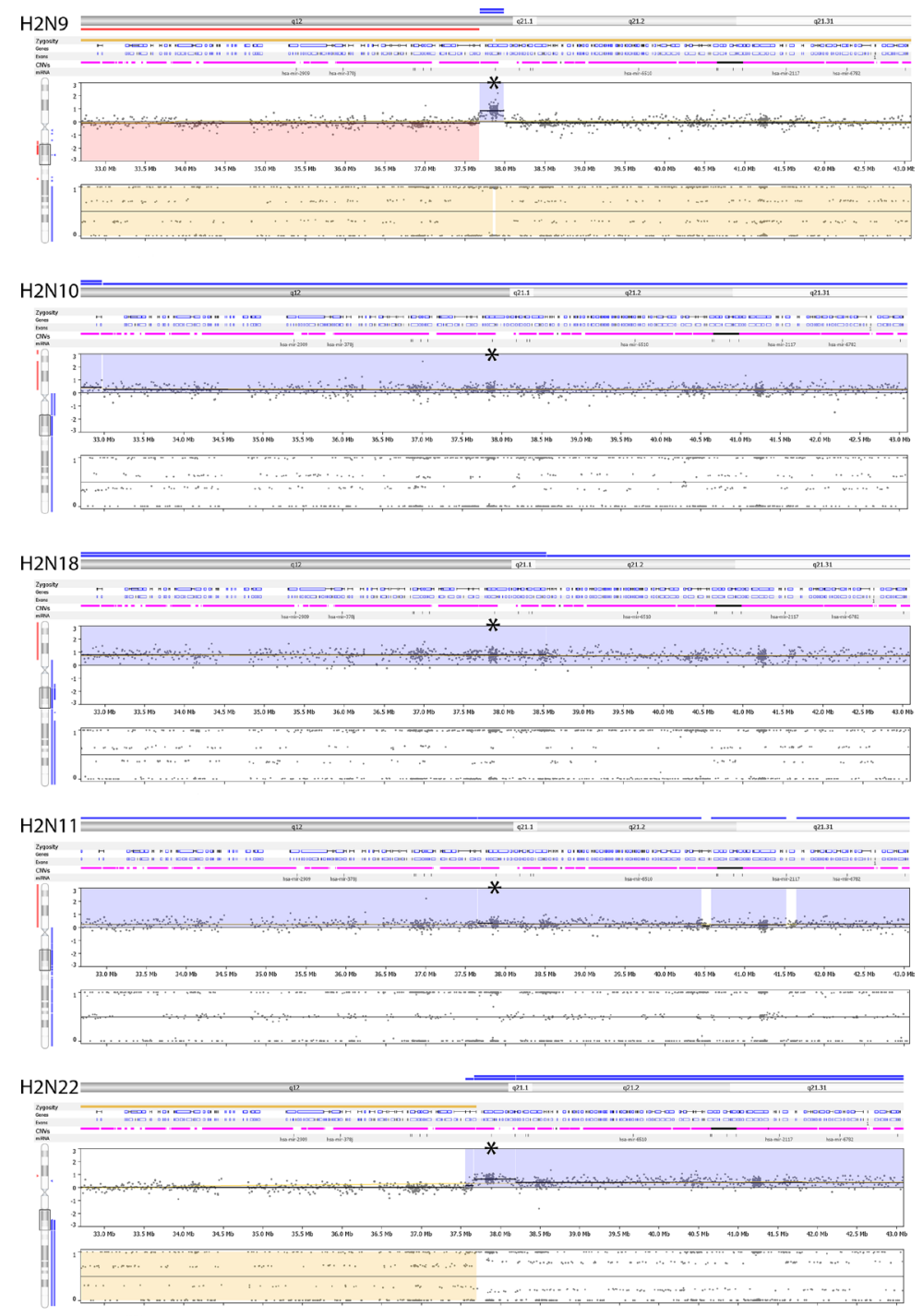

* ERBB2

Figure 8: Nexus screenshots of HER2-positive specimens ran on OncoScan. Only two (H2N9 and H2N22) out of the five have a focal amplification and one of them is adjacent to a large event $(\mathrm{H} 2 \mathrm{~N} 22-\mathrm{ERBB} 2$ copy number is 6 , which is flanked by normal $(\mathrm{CN}=2)$ and amplified $(\mathrm{CN}=4)$ regions. 
DNA was extracted from tumor and normal tissue FFPE blocks using the Qiagen GeneRead FFPE DNA extraction kit (Qiagen, Santa Clarita, CA), which has a repair step to minimize $\mathrm{C}$ to $\mathrm{T}$ transitions by removing deanimated cytosines. NGS libraries were constructed according to the protocol outlined by KAPA Biosystems. Briefly, DNA was sheared to an average size of 250-350 bp using sonication (Covaris). DNA fragment ends were repaired and phosphorylated using Klenow, T4 DNA Polymerase, and T4 Polynucleotide Kinase. An 'A' base was added to the 3' end of the blunted fragments, followed by ligation of single-indexed adapters via T-A mediated ligation. The library was PCR-amplified using 8 cycles, and tumor and normal libraries were pooled together at a 20:1 ratio (228ng tumor sample library and $12 \mathrm{ng}$ matched control). Five Tumor/Normal libraries were pooled together prior to capture and incubated with IDT xGen Lockdown probes and blocking oligos for 16-24 hours at $65^{\circ} \mathrm{C}$. Captured DNA was washed, eluted and PCR amplified using 10 cycles. The size and concentration of the amplified captured DNA library were determined using the Agilent TapeStation (Agilent Technologies, Santa Clara, CA). All samples were sequenced on an Illumina HiSeq2500, which generated $100 \times 100$ paired-end reads.

\section{Bioinformatic analysis}

Demultiplexing by barcode and sequence quality filtering was done in the Illumina Consensus Assessment of Sequence and Variation (CASAVA) software (v.1.8.2, Illumina, Hayward, CA). A custom bioinformatics pipeline was developed to perform paired analysis of tumor and germline DNA. Briefly, FASTQ files from CASAVA were aligned to the hg19 version of the human genome using Novoalign V3.02.07. Next, paired-sample analysis was performed using VarScan2 (v2.3.8). For both SNP and indel calling by VarScan2, the minimum variant frequency was set to $1 \%$ and the minimum coverage in tumor and normal was set to $6 \times$ and $4 \times$ respectively. Optimized variant calling filters were set at a read coverage of $\geq 100 \times$ for tumor DNA and $\geq 10 \times$ for matched control. Gene fusions including deletions, tandem duplications, inversions and translocations were detected using DELLY (v0.6.1). The minimum pair-end mapping quality was set at 1 while other settings were set at default. DELLY filtering required at least 3 paired-end reads supporting the fusion and paired-end mapping quality $\geq 20$. SV calls by DELLY were filtered out if determined to be due to repetitive regions. Paired normal samples were also analyzed using a custom bioinformatics pipeline that utilizes Novoalign V3.02.07 to align FASTQ reads to a reference sequence (hg19) and GATK (V3.2.2) to generate variants and no/low coverage reports. Germline variants were filtered using a Q score of $\geq 30$, coverage of $\geq 10 \times$, het ratio of $\geq 10 \%$ and filtered out if determined to be a sequencing artifact or common polymorphism utilizing population frequency data from multiple sources including NCBI dbSNP, NHLBI Exome Sequencing Project (ESP), 1000 Genomes, and internal Ambry data. Known causative variants outside reportable range are also protected from filtering. For quality control, the pipeline generates coverage metrics including: 1) number of total read pairs, 2) \% of mapped read pairs, 3) \% of PCR duplicates, 4) number of on-target read-pairs, 5) average coverage in target region, 6) target specificity and 7) $\%$ of bases at $\geq 10 \times, \geq 20 \times, \geq 50 \times, \geq$ $100 \times, \geq 200 \times, \geq 500 \times$, and $\geq 1000 \times$.

Annotation of variants was performed using custom scripts based on recommendations from the Human Genome Variation Society (HGVS). Gene fusions were annotated using Oncofuse v1.0.7 (ref: doi: 10.1093/ bioinformatics/btt445) to report only gene fusions where one of the two fusion partners is in our target genes.

The TumorNext pipeline was designed to achieve maximum sensitivity in detecting somatic variants in tumor samples whether matched control samples are available or not. In tumor-normal analysis mode, we applied Varscan2 (v2.3.6), a highly sensitive, heuristic based algorithm to detect somatic variants as low as 3\% minor allele frequency. Current efforts are focused on developing custom filters to remove low confidence calls with evidence from literature and public repositories. For structural variants, we applied DELLY v.0.6.1 to identify potential breakpoints supported by both paired-end and split reads from tumor sequence data.

\section{OncoScan}

The OncoScan workflow is based on the hybridization of MIPs to FFPE DNA samples and subsequent circularization, amplification and labeling. The labeled MIPs are hybridized to the OncoScan array, washed and scanned. The assays were set up according to the OncoScan sample preparation manual (P/N 703175 Rev. 1) using DNA isolated from FFPE tumor specimens using the Qiagen GeneRead FFPE DNA extraction kit (Qiagen, Santa Clarita, CA). Briefly, DNA samples are normalized to $12 \mathrm{ng} / \mu \mathrm{L}$, mixed with MIPs and incubated overnight to anneal (16-18 hours). Next, each reaction was divided equally into A and B reactions and "Gap Fill" master mix was added with either AT dNTPs (A reaction) or GC dNTPs (B reaction) and incubated. Following the "Gap Fill" reaction, exonuclease was added to remove unligated probes and genomic DNA. Next, MIPs were linearized with a restriction enzyme and PCR amplified (PCR 1). Reactions were taken through a second round of amplification (PCR 2) and subsequently digested with HaeIII restriction enzyme. The digested products were hybridized to the OncoScan Array for 16-18 hrs. Arrays were stained and washed using the GeneChip ${ }^{\circledR}$ Fluidics Station 450 and loaded on the GeneChip ${ }^{\circledR}$ Scanner 3000 7G (Affymetrix, Santa Clara, CA) where fluorescence intensity was scanned to generate array 
images (DAT files). Next, array fluorescence intensity data (CEL) files were generated and used to produce OSCHPTuScan files with the OncoScan ${ }^{\circledR}$ Console software version 1.1 using the reference files OncoScan.FFPE.n33. r1.REF MODEL for CNVs and OncoScan.FFPE.n33. r1.SOM REF MODEL for SNPs.

The TuScan algorithm is based on the ASCAT (allele-specific copy number analysis of tumors) algorithm, which determines allele specific copy number and simultaneously estimates and adjusts for both percent tumor and ploidy [49]. It provides copy number in $\log 2$ and linear scale, which can be viewed in Nexus Express for OncoScan. OncoScan uses the $\log R$ and BAF to determine copy number. The $\log \mathrm{R}$ ratio is the logged ratio of observed probe intensity to the expected intensity - any deviations from zero indicate copy number change. BAF allows detection of allelic imbalance. A value near 0.5 indicates a heterozygous genotype $(\mathrm{AB})$, whereas 0 and 1 indicate a homozygous genotype ( $\mathrm{AA}$ and $\mathrm{BB})-$ in a normal diploid sample, there is a mix of $\mathrm{AA}, \mathrm{AB}$ and $\mathrm{BB}$ genotypes. Deletions, copy neutral loss of heterozygosity, imbalanced amplifications and mosaic samples exhibit altered BAF plots. Unlike algorithms that use uniform thresholds, TuScan can detect CNVs when only present in a minority of cells as the algorithm determines what deviations from $\log \mathrm{R}$ and $\mathrm{BAF}$ are consistent with the percent tumor and ploidy of the sample.

\section{ACKNOWLEDGMENTS AND FUNDING}

The authors would like to acknowledge Hansook Chong, Shela Lee, Cody Buhler and Shuwei Li from Ambry Genetics and Jonathan Foos from Affymetrix for technical advice, and Emmanuelle Nicolas and Ryan Winter from Fox Chase Cancer Center for support with specimens. FCCC authors were supported by NCI Core Grant P30 CA006927.

\section{CONFLICTS OF INTEREST}

PG, HV, PT, HL, WM, VH, JH, SS, LU, SF, HP and AE are all employees of Ambry Genetics. MJ is a paid consultant for Ambry Genetics.

\section{REFERENCES}

1. Igbokwe A, Lopez-Terrada DH. Molecular testing of solid tumors. Arch Pathol Lab Med. 2011; 135:67-82.

2. McCourt CM, Boyle D, James J, Salto-Tellez M. Immunohistochemistry in the era of personalised medicine. J Clin Pathol. 2013; 66:58-61.

3. Pauletti G, Dandekar S, Rong H, Ramos L, Peng H, Seshadri R, Slamon DJ. Assessment of methods for tissuebased detection of the HER-2/neu alteration in human breast cancer: a direct comparison of fluorescence in situ hybridization and immunohistochemistry. J Clin Oncol. 2000; 18:3651-3664.

4. Lebeau A, Deimling D, Kaltz C, Sendelhofert A, Iff A, Luthardt B, Untch M, Lohrs U. Her-2/neu analysis in archival tissue samples of human breast cancer: comparison of immunohistochemistry and fluorescence in situ hybridization. J Clin Oncol. 2001; 19:354-363.

5. Angulo B, Garcia-Garcia E, Martinez R, Suarez-Gauthier A, Conde E, Hidalgo M, Lopez-Rios F. A commercial real-time PCR kit provides greater sensitivity than direct sequencing to detect KRAS mutations: a morphology-based approach in colorectal carcinoma. J Mol Diagn. 2010; 12:292-299.

6. Gonzalez de Castro D, Angulo B, Gomez B, Mair D, Martinez R, Suarez-Gauthier A, Shieh F, Velez M, Brophy VH, Lawrence HJ, Lopez-Rios F. A comparison of three methods for detecting KRAS mutations in formalinfixed colorectal cancer specimens. Br J Cancer. 2012; 107:345-351.

7. Lopez-Rios F, Angulo B, Gomez B, Mair D, Martinez R, Conde E, Shieh F, Vaks J, Langland R, Lawrence HJ, de Castro DG. Comparison of testing methods for the detection of BRAF V600E mutations in malignant melanoma: preapproval validation study of the companion diagnostic test for vemurafenib. PLoS One. 2013; 8:e53733.

8. Solin LJ, Gray R, Baehner FL, Butler SM, Hughes LL, Yoshizawa C, Cherbavaz DB, Shak S, Page DL, Sledge GW, Jr, Davidson NE, Ingle JN, et al. A multigene expression assay to predict local recurrence risk for ductal carcinoma in situ of the breast. J Natl Cancer Inst. 2013; 105:701-710.

9. Wagle N, Berger MF, Davis MJ, Blumenstiel B, Defelice M, Pochanard P, Ducar M, Van Hummelen P, Macconaill LE, Hahn WC, Meyerson M, Gabriel SB, Garraway LA. Highthroughput detection of actionable genomic alterations in clinical tumor samples by targeted, massively parallel sequencing. Cancer Discov. 2011; 2:82-93.

10. Frampton GM, Fichtenholtz A, Otto GA, Wang K, Downing SR, He J, Schnall-Levin M, White J, Sanford EM, An P, Sun J, Juhn F, Brennan K, et al. Development and validation of a clinical cancer genomic profiling test based on massively parallel DNA sequencing. Nat Biotechnol. 2013; 31:1023-1031

11. Cottrell CE, Al-Kateb H, Bredemeyer AJ, Duncavage EJ, Spencer DH, Abel HJ, Lockwood CM, Hagemann IS, O'Guin SM, Burcea LC, Sawyer CS, Oschwald DM, Stratman JL, et al. Validation of a next-generation sequencing assay for clinical molecular oncology. J Mol Diagn. 2014; 16:89-105.

12. Pritchard CC, Salipante SJ, Koehler K, Smith C, Scroggins S, Wood B, Wu D, Lee MK, Dintzis S, Adey A, Liu Y, Eaton KD, Martins R, et al. Validation and implementation of targeted capture and sequencing for the detection of actionable mutation, copy number variation, and gene rearrangement in clinical cancer specimens. J Mol Diagn. 2014; 16:56-67. 
13. "Next Generation" Sequencing (NGS) guidelines for somatic genetic variant detection. 2015 .

14. Mamanova L, Coffey AJ, Scott CE, Kozarewa I, Turner EH, Kumar A, Howard E, Shendure J, Turner DJ. Targetenrichment strategies for next-generation sequencing. Nat Methods. 2010; 7:111-118.

15. Smith EN, Jepsen K, Khosroheidari M, Rassenti LZ, D'Antonio M, Ghia EM, Carson DA, Jamieson $\mathrm{CH}$, Kipps TJ, Frazer KA. Biased estimates of clonal evolution and subclonal heterogeneity can arise from PCR duplicates in deep sequencing experiments. Genome Biol. 2014; 15:420.

16. Jones S, Anagnostou V, Lytle K, Parpart-Li S, Nesselbush M, Riley DR, Shukla M, Chesnick B, Kadan M, Papp E, Galens KG, Murphy D, Zhang T, et al. Personalized genomic analyses for cancer mutation discovery and interpretation. Sci Transl Med. 2015; 7:283ra253.

17. Hodgson S. Mechanisms of inherited cancer susceptibility. J Zhejiang Univ Sci B. 2008; 9:1-4.

18. Parsons DW, Roy A, Plon SE, Roychowdhury S, Chinnaiyan AM. Clinical tumor sequencing: an incidental casualty of the American College of Medical Genetics and Genomics recommendations for reporting of incidental findings. J Clin Oncol. 2014; 32:2203-2205.

19. Pinkel D, Segraves R, Sudar D, Clark S, Poole I, Kowbel D, Collins C, Kuo WL, Chen C, Zhai Y, Dairkee SH, Ljung BM, Gray JW, et al. High resolution analysis of DNA copy number variation using comparative genomic hybridization to microarrays. Nat Genet. 1998; 20:207-211.

20. Pollack JR, Perou CM, Alizadeh AA, Eisen MB, Pergamenschikov A, Williams CF, Jeffrey SS, Botstein D, Brown PO. Genome-wide analysis of DNA copy-number changes using cDNA microarrays. Nat Genet. 1999; 23:41-46.

21. Hagenkord JM, Chang CC. The rewards and challenges of array-based karyotyping for clinical oncology applications. Leukemia. 2009; 23:829-833.

22. Gunn SR, Mohammed MS, Gorre ME, Cotter PD, Kim J, Bahler DW, Preobrazhensky SN, Higgins RA, Bolla AR, Ismail $\mathrm{SH}$, de Jong $\mathrm{D}$, Eldering $\mathrm{E}$, van Oers $\mathrm{MH}$, et al. Whole-genome scanning by array comparative genomic hybridization as a clinical tool for risk assessment in chronic lymphocytic leukemia. J Mol Diagn. 2008; 10:442-451.

23. Lyons-Weiler M, Hagenkord J, Sciulli C, Dhir R, Monzon FA. Optimization of the Affymetrix GeneChip Mapping 10K 2.0 Assay for routine clinical use on formalin-fixed paraffinembedded tissues. Diagn Mol Pathol. 2008; 17:3-13.

24. Lo J, Kerns BJ, Amling CL, Robertson CN, Layfield LJ. Correlation of DNA ploidy and histologic diagnosis from prostate core-needle biopsies: is DNA ploidy more sensitive than histology for the diagnosis of carcinoma in small specimens? J Surg Oncol. 1996; 63:41-45.

25. Koivisto P. Aneuploidy and rapid cell proliferation in recurrent prostate cancers with androgen receptor gene amplification. Prostate Cancer Prostatic Dis. 1997; 1:21-25.
26. Reinholz MM, Bruzek AK, Visscher DW, Lingle WL, Schroeder MJ, Perez EA, Jenkins RB. Breast cancer and aneusomy 17: implications for carcinogenesis and therapeutic response. The Lancet. 2009; 10:267-277.

27. Krishnamurti U, Hammers JL, Atem FD, Storto PD, Silverman JF. Poor prognostic significance of unamplified chromosome 17 polysomy in invasive breast carcinoma. Mod Pathol. 2009; 22:1044-1048.

28. Watters AD, Going JJ, Cooke TG, Bartlett JM. Chromosome 17 aneusomy is associated with poor prognostic factors in invasive breast carcinoma. Breast Cancer Res Treat. 2003; 77:109-114.

29. Mehta S, Shelling A, Muthukaruppan A, Lasham A, Blenkiron C, Laking G, Print C. Predictive and prognostic molecular markers for cancer medicine. Ther Adv Med Oncol. 2010; 2:125-148.

30. Heppner GH. Tumor heterogeneity. Cancer research. 1984; 44:2259-2265.

31. Marusyk A, Polyak K. Tumor heterogeneity: causes and consequences. Biochimica et biophysica acta. 2010; 1805:105-117.

32. Lauring AS, Andino R. Quasispecies theory and the behavior of RNA viruses. PLoS Pathog. 2010; 6:e1001005.

33. Shah SP, Morin RD, Khattra J, Prentice L, Pugh T, Burleigh A, Delaney A, Gelmon K, Guliany R, Senz J, Steidl C, Holt RA, Jones S, et al. Mutational evolution in a lobular breast tumour profiled at single nucleotide resolution. Nature. 2009; 461:809-813.

34. Cheng DT, Mitchell TN, Zehir A, Shah RH, Benayed R, Syed A, Chandramohan R, Liu ZY, Won HH, Scott SN, Brannon AR, O’Reilly C, Sadowska J, et al. Memorial Sloan Kettering-Integrated Mutation Profiling of Actionable Cancer Targets (MSK-IMPACT): A Hybridization Capture-Based Next-Generation Sequencing Clinical Assay for Solid Tumor Molecular Oncology. J Mol Diagn. 2015; 17:251-264.

35. Samuels Y, Wang Z, Bardelli A, Silliman N, Ptak J, Szabo S, Yan H, Gazdar A, Powell SM, Riggins GJ, Willson JK, Markowitz S, Kinzler KW, et al. High frequency of mutations of the PIK3CA gene in human cancers. Science (New York, NY. 2004; 304:554.

36. Bose R, Kavuri SM, Searleman AC, Shen W, Shen D, Koboldt DC, Monsey J, Goel N, Aronson AB, Li S, Ma CX, Ding L, Mardis ER, et al. Activating HER2 mutations in HER2 gene amplification negative breast cancer. Cancer Discov. 2013; 3:224-237.

37. Wang Y, Carlton VE, Karlin-Neumann G, Sapolsky R, Zhang L, Moorhead M, Wang ZC, Richardson AL, Warren R, Walther A, Bondy M, Sahin A, Krahe R, et al. High quality copy number and genotype data from FFPE samples using Molecular Inversion Probe (MIP) microarrays. BMC Med Genomics. 2009; 2:8.

38. Wolff AC, Hammond ME, Hicks DG, Dowsett M, McShane LM, Allison KH, Allred DC, Bartlett JM, Bilous M, Fitzgibbons P, Hanna W, Jenkins RB, Mangu PB, et al. Recommendations for human epidermal growth 
factor receptor 2 testing in breast cancer: American Society of Clinical Oncology/College of American Pathologists clinical practice guideline update. J Clin Oncol. 2013; 31:3997-4013.

39. Gunn S, Yeh IT, Lytvak I, Tirtorahardjo B, Dzidic N, Zadeh S, Kim J, McCaskill C, Lim L, Gorre M, Mohammed M. Clinical array-based karyotyping of breast cancer with equivocal HER2 status resolves gene copy number and reveals chromosome 17 complexity. BMC Cancer. 2010; 10:396.

40. Sapino A, Goia M, Recupero D, Marchio C. Current Challenges for HER2 Testing in Diagnostic Pathology: State of the Art and Controversial Issues. Front Oncol. 2013; 3:129.

41. Schwaederle M, Daniels GA, Piccioni DE, Fanta PT, Schwab RB, Shimabukuro KA, Parker BA, Kurzrock R. Cyclin alterations in diverse cancers: Outcome and coamplification network. Oncotarget. 2015; 6:3033-3042. doi: 10.18632/oncotarget.2848.

42. Knudson AG, Jr. Mutation and cancer: statistical study of retinoblastoma. Proc Natl Acad Sci USA. 1971; 68:820-823.

43. Knudson AG. Two genetic hits (more or less) to cancer. Nat Rev Cancer. 2001; 1:157-162.

44. Grasso CS, Wu YM, Robinson DR, Cao X, Dhanasekaran SM, Khan AP, Quist MJ, Jing X, Lonigro RJ, Brenner JC, Asangani IA, Ateeq B, Chun SY, et al. The mutational landscape of lethal castration-resistant prostate cancer. Nature. 2012; 487:239-243.
45. Mateo J, Carreira S, Sandhu S, Miranda S, Mossop H, Perez-Lopez R, Nava Rodrigues D, Robinson D, Omlin A, Tunariu N, Boysen G, Porta N, Flohr P, et al. DNA-Repair Defects and Olaparib in Metastatic Prostate Cancer. N Engl J Med. 2015; 373:1697-1708.

46. Pennington KP, Walsh T, Harrell MI, Lee MK, Pennil CC, Rendi MH, Thornton A, Norquist BM, Casadei S, Nord AS, Agnew KJ, Pritchard CC, Scroggins S, et al. Germline and somatic mutations in homologous recombination genes predict platinum response and survival in ovarian, fallopian tube, and peritoneal carcinomas. Clin Cancer Res. 2014; 20:764-775.

47. Ciriello G, Miller ML, Aksoy BA, Senbabaoglu Y, Schultz N, Sander C. Emerging landscape of oncogenic signatures across human cancers. Nat Genet. 2013; 45:1127-1133.

48. Green RC, Berg JS, Grody WW, Kalia SS, Korf BR, Martin CL, McGuire A, Nussbaum RL, O'Daniel JM, Ormond KE, Rehm HL, Watson MS, Williams MS, et al. ACMG Recommendations for Reporting of Incidental Findings in Clinical Exome and Genome Sequencing. Genet Med. 2013; 15:565-574.

49. Van Loo P, Nordgard SH, Lingjaerde OC, Russnes HG, Rye IH, Sun W, Weigman VJ, Marynen P, Zetterberg A, Naume B, Perou CM, Borresen-Dale AL, Kristensen VN. Allele-specific copy number analysis of tumors. Proc Natl Acad Sci USA. 2010; 107:16910-16915. 